Gut, 1960, 1, 149.

\title{
PARTIAL GASTRECTOMY FOR PEPTIC ULCER
}

\section{BY}

\author{
VICTOR S. BROOKES, J. A. H. WATERHOUSE, and P. A. THORN
}

From the University of Birmingham, the United Birmingham Hospitals, and Wolverhampton and

\section{Kidderminster Hospitals}

This paper reports a study of 955 patients in whom partial gastrectomy was performed for peptic ulcer. An analysis is presented of the clinical and operative factors which may influence the success of the operation.

In a recent investigation undertaken by us to determine the incidence of pulmonary tuberculosis in patients who had undergone partial gastrectomy for ulcer (Thorn, Brookes, and Waterhouse, 1956), the opportunity was taken to collect as much information as possible regarding the results of gastrectomy for ulcer. An attempt is made in this paper to assess these results, and to analyse the factors influencing them.

\section{MATERIAL}

The information collected concerned all patients in whom a gastrectomy for peptic ulcer was carried out by the surgeons attached to the Surgical Pro- fessorial Unit of Birmingham University (at the Queen Elizabeth Hospital) between the beginning of 1948 and the middle of 1953 . In addition to the information available from previous interviews at a routine gastrectomy follow-up clinic, two of us (B and T) further interviewed and examined as many of these patients as could be traced. In a small number, where personal interview was not possible, information was collected by questionnaire.

The figures obtained were analysed with the aid of a punch card system under the supervision of the Medical Statistics Department of Birmingham University (W).

Cases were not selected for the investigation and the series includes all those operated on as emer-

TABLE I

SITE OF ULCER, SEX, AND YEAR OF PARTIAL GASTRECTOMY

\begin{tabular}{|c|c|c|c|c|c|c|c|c|c|c|c|c|c|c|}
\hline \multirow{2}{*}{ Site } & \multicolumn{7}{|c|}{ Males } & \multicolumn{7}{|c|}{ Females } \\
\hline & 1948 & 1949 & 1950 & 1951 & 1952 & $1953\left(\frac{1}{2}\right)$ & Total (\%) & 1948 & 1949 & 1950 & 1951 & 1952 & $1953\left(\frac{1}{2}\right)$ & Total (\%) \\
\hline $\begin{array}{l}\text { Gastric } \\
\text { Duodenal } \\
\text { Gastric plus duodenal } \\
\text { Other sites* }\end{array}$ & $\begin{array}{r}42 \\
47 \\
5 \\
2\end{array}$ & $\begin{array}{r}46 \\
102 \\
6 \\
4\end{array}$ & $\begin{array}{l}39 \\
87 \\
14 \\
13\end{array}$ & $\begin{array}{r}41 \\
94 \\
7 \\
7\end{array}$ & $\begin{array}{r}49 \\
85 \\
11 \\
4\end{array}$ & $\begin{array}{r}23 \\
36 \\
4 \\
4\end{array}$ & $\begin{array}{c}240(30.9) \\
451(58 \cdot 4) \\
47(6 \cdot 1) \\
34(4 \cdot 5)\end{array}$ & $\begin{array}{l}8 \\
9 \\
4 \\
1\end{array}$ & $\begin{array}{l}19 \\
15 \\
-\end{array}$ & $\begin{array}{r}11 \\
16 \\
3 \\
-\end{array}$ & $\begin{array}{r}12 \\
25 \\
1 \\
-\end{array}$ & $\begin{array}{r}19 \\
22 \\
3 \\
2\end{array}$ & $\begin{array}{l}\frac{10}{3} \\
=\end{array}$ & $\begin{array}{c}79(43 \cdot 3) \\
90(49 \cdot 2) \\
11(6 \cdot 0) \\
3(1 \cdot 6)\end{array}$ \\
\hline Total & 96 & 158 & 153 & 149 & 149 & 67 & 772 & 22 & 34 & 30 & 38 & 46 & 13 & 183 \\
\hline
\end{tabular}

*Other sites includes stomal ulcer after gastroenterostomy and prolapse of gastric mucous membrane through pyloric ring.

TABLE II

AGE AT TIME OF PARTIAL GASTRECTOMY

\begin{tabular}{|c|c|c|c|c|c|c|}
\hline \multirow{3}{*}{ Age in Years } & \multicolumn{3}{|c|}{ Males } & \multicolumn{3}{|c|}{ Females } \\
\hline & All Sites & Gastric & Duodenal & All Sites & Gastric & Duodenal \\
\hline & No. & No. & No. & No. & No. & No. \\
\hline $\begin{array}{l}\text { Under } 25 \\
255-34 \\
35-44 \\
45-54 \\
555-64 \\
65 \text { and over }\end{array}$ & $\begin{array}{c}14(0.8 \%) \\
80(10.3 \%) \\
186(24.1 \%) \\
289(37.5 \%) \\
159(20.6 \%) \\
44(5.7 \%)\end{array}$ & $\begin{array}{c}9 \overline{(3.8 \%)} \\
54(22.6 \%) \\
96(39.7 \%) \\
63(26.4 \%) \\
18(7.5 \%)\end{array}$ & $\begin{array}{c}12(2.7 \%) \\
64(14.2 \%) \\
115(25.5 \%) \\
164(36.3 \%) \\
77(17.1 \%) \\
19(4.2 \%)\end{array}$ & $\begin{array}{l}3(1.6 \%) \\
20(10.9 \%) \\
39(21.3 \%) \\
54(29.5 \%) \\
53(29.0 \%) \\
14(7.7 \%)\end{array}$ & $\begin{array}{r}1(1.3 \%) \\
8(10 \cdot 1 \%) \\
10(12 \cdot 7 \%) \\
22(27.8 \%) \\
30(38.0 \%) \\
8(10 \cdot 1 \%)\end{array}$ & $\begin{array}{l}2(2 \cdot 2 \%) \\
12(13.3 \%) \\
27(30.0 \%) \\
28(31.1 \%) \\
19(21.1 \%) \\
2(2.2 \%)\end{array}$ \\
\hline Total & 772 & 240 & 451 & 183 & 79 & 90 \\
\hline
\end{tabular}

N.B.-In this table the group with both gastric and duodenal ulcer and the group classed as "other sites" are not analysed because they are relatively small. 
gencies, the majority of the latter having been operated on for haematemesis or melaena.

The number of patients considered in this review is 955 and the distribution of these according to sex, age, and site of ulcer, is shown in Tables I and II.

In duodenal ulcer the average age for men was 45.9 years and for women 45.8 years, while in gastric ulcer it was 50.6 years for men and 51.6 years for women.

The proportion of duodenal to gastric ulcer is 1.7:1, the percentage of gastric ulcers in this series being rather higher than in most other series.

\section{CASES Followed Up}

Of the total of 955 patients, 79 were known to be dead at the time of the follow-up investigation and of the remaining 876 the number available for assessment by us was $826(94.3 \%)$ (Table III). Some
TABLE IV

MORTALITY RATES ACCORDING TO AGE

\begin{tabular}{l|c|c|c}
\hline \multicolumn{1}{c|}{ Age (years) } & Total Cases & Died & Mortality Rate \% \\
\cline { 2 - 4 } Under 44 & 342 & 5 & 1.46 \\
$45-54$ & 343 & 10 & 2.92 \\
$55-64$ & 212 & 14 & $6 \cdot 6$ \\
Over 65 & 58 & 8 & 13.8 \\
\hline
\end{tabular}

recovery from operation, was $3.87 \%$, but it showed a considerable variation according to age (Table IV).

While deaths due to technical failures (10 cases, $1.05 \%$ ) were spread evenly over all age groups, those due to cardiovascular (nine cases, $0.84 \%$ ) and respiratory causes (10 cases, $1.05 \%$ ) accounted for the great increase in mortality in the older patients.

ASSESSMENT OF the Results of Gastrectomy

In the investigation carried out by two of us (B and T) many aspects of the result of gastrectomy were considered. These included the relative

TABLE III

DEATHS AND NUMBERS OF CASES FOLLOWED UP ACCORDING TO SEX AND SITE OF ULCER

\begin{tabular}{|c|c|c|c|c|c|c|c|c|}
\hline & $\begin{array}{c}\text { Total } \\
\text { Operated }\end{array}$ & $\begin{array}{c}\text { Operation } \\
\text { Deaths }\end{array}$ & $\begin{array}{c}\text { Late } \\
\text { Gastrectomy }\end{array}$ & $\begin{array}{l}\text { Pulmonary } \\
\text { Tuberculosis }\end{array}$ & $\begin{array}{c}\text { Other } \\
\text { Diseases and } \\
\text { Accidents }\end{array}$ & $\begin{array}{c}\text { Total } \\
\text { Deaths }\end{array}$ & $\begin{array}{c}\text { Not } \\
\text { Followed } \\
\text { Up }\end{array}$ & $\begin{array}{l}\text { Followed } \\
\text { Up }\end{array}$ \\
\hline $\begin{array}{l}\text { Duodenal ulcer (men) } \\
\text { Duodenal ulcer (women) } \\
\text { Gastric ulcer (men) } \\
\text { Gastric ulcer (women) } \\
\text { Duodenal and gastric ulcer (men) } \\
\text { Duodenal and gastric ulcer (women) } \\
\text { Others (men) } \\
\text { Others (women) }\end{array}$ & $\begin{array}{r}451 \\
90 \\
240 \\
79 \\
47 \\
11 \\
34 \\
3\end{array}$ & $\begin{array}{l}16(3.5 \%) \\
2(2 \cdot 2 \%) \\
9(3.8 \%) \\
3(3.8 \%) \\
3(6.4 \%) \\
0(0 \%) \\
4(11.8 \%) \\
0(0 \%)\end{array}$ & $\begin{array}{l}2 \\
0 \\
1 \\
0 \\
2 \\
0 \\
0 \\
0\end{array}$ & $\begin{array}{l}\mathbf{0} \\
\mathbf{0} \\
\mathbf{7} \\
\mathbf{1} \\
\mathbf{0} \\
\mathbf{0} \\
\mathbf{0} \\
\mathbf{0}\end{array}$ & $\begin{array}{r}9 \\
1 \\
10 \\
7 \\
1 \\
0 \\
1 \\
0\end{array}$ & $\begin{array}{c}27 \\
3 \\
27 \\
11 \\
6 \\
0 \\
5 \\
0\end{array}$ & $\begin{array}{l}21(4.7 \%) \\
5(5.6 \%) \\
14(5.8 \%) \\
3(3.8 \%) \\
4(8.6 \%) \\
1(9.1 \%) \\
2(5.9 \%) \\
0(0 \%)\end{array}$ & $\begin{array}{r}403 \\
82 \\
199 \\
65 \\
37 \\
10 \\
27 \\
3\end{array}$ \\
\hline Total (men) & 772 & $32(4 \cdot 1 \%)$ & 5 & 7 & 21 & 65 & $41(5.3 \%)$ & 666 \\
\hline Total (women) & 183 & $5(2.7 \%)$ & 0 & 1 & 8 & 14 & $9(4.9 \%)$ & 160 \\
\hline Grand total & 955 & $37(3.9 \%)$ & 5 & 8 & 29 & 79 & $50(5.2 \%)$ & 826 \\
\hline
\end{tabular}

of the patients not traced and also some of those who had died some considerable time after operation had been seen at some time previously in the routine follow-up clinic and some assessment of the results of their operations had been made. Where this is appropriate such information will be given in this paper.

\section{DEATHS}

Of the 79 patients known to be dead, 37 could be classified in the group "operative mortality", while a further five were styled "late gastrectomy deaths" since they died of late complications attributable to partial gastrectomy. Eight patients died of pulmonary tuberculosis which we considered was probably related to the gastrectomy (Thorn et al., 1956). The remaining 29 died of other diseases unassociated with the operation.

The operative mortality rate, which included all patients who died before making a complete amounts of work lost per year before and after operation, earning capacity, change of occupation, post-gastrectomy symptoms, bowel habits following gastrectomy, pre- and post-operative body weights and their comparison with standard weights, evidence of gastrointestinal haemorrhage before and after operation, post-operative anaemia (including haemoglobin estimation at our interview), and chest radiograph. A final assessment, in which the patient's own assessment of his or her condition was taken into account, was made of the result of the operation in each case seen. Where opinions conflicted the lower or poorer estimate of the result is the one given.

We assessed results according to five basic groups. These, over all, were similar to the groups or grades used by Visick (1948a), but we adapted them slightly so that the patients, in making their own assessment, could clearly decide which group they considered they were in. 
These five groups were as follows:-

Group 1.-Completely symptom-free and able to lead a normal life.

Group 2.-Mild symptoms needing no dietary care or any other treatment and not interfering with normal life.

Group 3.-Mild symptoms which need dietary care or other treatment but not sufficient to interfere with normal life.

Group 4.-Symptoms, which, or the care of which, interfere with normal life but where the patient on the whole is better than he was before operation.

Group 5.-Symptoms as a result of which the patient is neither better nor worse than before operation.

In order to obtain groups large enough to give figures of statistical significance, we have added groups 1 and 2 together and called them "satisfactory results" and groups 4 and 5 together and called them "unsatisfactory results".

In all instances the assessment given is that of the original gastrectomy. In some cases subsequent operations have been performed, for example, refashioning of gastrectomy, vagotomy, enteroanastomosis, which have relieved the patient of his or her post-gastrectomy symptoms and have correspondingly improved the result. Such cases, in our review of the original operation, are still classified in group 5. Symptoms or the necessity for medical treatment suggesting a recurrent ulcer, even where no further surgery has been done and even if the patient's condition is satisfactory on such a medical régime, have similarly been placed in group 5. On this basis 826 cases have been classified according to sex and ulcer site and the results are given in Table V. Over all we may say that the 540 patients in groups 1 and $2(65.4 \%$ of the cases followed up) were satisfactory, and needed no further treatment, and that the 727 in groups 1, 2, and $3(88 \%)$ were able to lead a more normal life after the operation than beforehand. The remaining 99 patients, i.e., groups 4 and 5, were unable to lead a normal life after the operation, and of these $6.9 \%$, i.e., group 5 patients, were not improved at all or were even worse than before operation.

FACTORS Influencing Result.-In discussing these cases we have tried to determine if there was any significant factor or factors which influenced the result. We therefore present analyses in such a way that any such factors may become apparent. Although in most instances we have analysed and assessed all five groups of results individually, we shall compare two main groups: (a) the satisfactory results (groups 1 and 2) and (b) the unsatisfactory results (groups 4 and 5) and only where it may seem significant will the analyses of all five groups be given separately. Furthermore, in an attempt to obtain fairly large groups of similar cases we have taken four main sub-groups: (i) Male duodenal ulcer, (ii) female duodenal ulcer, (iii) male gastric ulcer, and (iv) female gastric ulcer. Other groups were so small when broken down that their numbers were of no significance when trying to determine causative factors.

TABLE V

RESULTS OF GASTRECTOMY

\begin{tabular}{|c|c|c|c|c|c|c|}
\hline & \multicolumn{5}{|c|}{ Group } & \multirow{2}{*}{ Total } \\
\hline & 1 & 2 & 3 & 4 & 5 & \\
\hline $\begin{array}{l}\text { Duodenal ulcer (men) } \\
\text { Duodenal ulcer (women) } \\
\text { Gastric ulcer (men) } \\
\text { Gastric ulcer (women) } \\
\text { Duodenal and gastric ulcer (men) } \\
\text { Duodenal and gastric ulcer (women) } \\
\text { Others (men) } \\
\text { Others (women) }\end{array}$ & $\begin{array}{c}119 \\
(29.5 \%) \\
17 \% \\
(20.7 \%) \\
63 \% \\
(31.7 \%) \\
15 \% \\
(23.1 \%) \\
10 \\
(27.0 \%) \\
1 \\
(10.0 \%) \\
6 \% \\
(22.2 \%) \\
2 \% \\
(66.7 \%) \\
\end{array}$ & $\begin{array}{c}158 \\
(39.2 \%) \\
30 \\
(36.6 \%) \\
71 \\
(35.7 \%) \\
22 \% \\
(33.8 \%) \\
13 \% \\
(35.1 \%) \\
2 \% \\
(20.0 \%) \\
11 \% \\
(40.7 \%) \\
0 \\
(0 \%) \\
\end{array}$ & $\begin{array}{c}74 \\
(18.4 \%) \\
27 \% \\
(32.9 \%) \\
42 \% \\
(21.1 \%) \\
21 \\
(32.3 \%) \\
11 \% \\
(29.7 \%) \\
5 \% \\
(50.0 \%) \\
6 \% \\
(22.2 \%) \\
1 \% \\
(33.3 \%) \\
\end{array}$ & $\begin{array}{c}17 \\
(4.2 \%) \\
3 \\
(3.7 \%) \\
13 \% \\
(6.5 \%) \\
5 \\
(7.7 \%) \\
1 \% \\
(2.7 \%) \\
2 \% \\
(20.0 \%) \\
1 \\
(3.7 \%) \\
0 \\
(0 \%) \\
\end{array}$ & $\begin{array}{c}35 \\
(8.7 \%) \\
5 \\
(6.1 \%) \\
10 \\
(5.0 \%) \\
2 \% \\
(3.1 \%) \\
2 \%) \\
(5.4 \%) \\
0 \\
(0 \%) \\
3 \\
(11.1 \%) \\
0 \\
(0 \%) \\
\end{array}$ & $\begin{array}{r}403 \\
82 \\
199 \\
65 \\
37 \\
10 \\
27 \\
3\end{array}$ \\
\hline Total (men) & $\begin{array}{c}198 \\
(29.7 \%)\end{array}$ & $\begin{array}{c}253 \\
(38.0 \%)\end{array}$ & $\begin{array}{c}133 \\
(20.0 \%)\end{array}$ & $\begin{array}{c}32 \\
(4 \cdot 8 \%)\end{array}$ & $\begin{array}{c}50 \\
(7.5 \%)\end{array}$ & 666 \\
\hline Total (women) & $\begin{array}{c}35 \\
(21.9 \%)\end{array}$ & $\begin{array}{c}54 \\
(33.8 \%)\end{array}$ & $\begin{array}{c}54 \\
(33.8 \%)\end{array}$ & $\begin{array}{c}10 \\
(6 \cdot 3 \%)\end{array}$ & $(4.4 \%)$ & 160 \\
\hline Grand total & $\begin{array}{c}233 \\
(28.2 \%)\end{array}$ & $\begin{array}{c}307 \\
(37 \cdot 2 \%)\end{array}$ & $\begin{array}{c}187 \\
(22.6 \%)\end{array}$ & $\begin{array}{c}42 \\
(5 \cdot 1 \%)\end{array}$ & $\begin{array}{c}57 \\
(6.9 \%)\end{array}$ & 826 \\
\hline
\end{tabular}


(1) Effect of Length of Follow-up on Result of Gastrectomy.-The results of operation in the four main sub-groups mentioned in the last paragraph were assessed against length of follow-up. Since the operations had been performed over a number of years the follow-up time varied from one to six years.

In the analyses the percentage of good results in the total of all the four sub-groups showed a progressive fall during the first four years after operation, but following this the percentages rose again. In the larger sub-groups the same trend is reflected, while in the smaller ones the figures were too small to be significant.

The probable explanation for this peculiar variation in the results is that while some causes of failure, such as recurrent ulcer, might slowly increase with time, other forms of post-gastrectomy symptoms, which may adversely affect the result, sometimes diminish with the passage of time. From the records available at the previous follow-up clinic it was apparent that 12 patients whom we saw and assessed as satisfactory had had, at some time previously, symptoms which would have made them unsatisfactory if we had seen them at that time. On the other hand nine patients whom we classed as unsatisfactory had been quite well for some considerable time (four cases for as long as four years) before deteriorating. This possible variation in the result from time to time illustrates the difficulty of assessing results against length of follow-up, and Visick (1948b) has also drawn attention to it.

However, since there is no particular trend for results to move gradually from group 1 to group 5 or vice versa, and also since the percentage results for each year group did not show any great variation from the average result for the total, we shall not, having pointed out these facts, subdivide the cases according to length of follow-up in our evaluations of other factors.

(2) Effect of Age on Result of Gastrectomy.There appears to be no significant difference in the results between the various age groups (Table VI) and in this series we conclude that age does not affect the result of gastrectomy.

(3) Effect of Site of Ulcer and of Sex on Results of Gastrectomy.-In an effort to determine whether sex or the original site of the ulcer affects the result of the gastrectomy, we have condensed the totals shown in Table VII from the previous results given.

As far as the satisfactory results are concerned there appears to be no difference between gastric and duodenal ulcer in either sex and this is reflected in the totals. There is a difference in the results, however, between male and female which is significant $(P>0.02)$ in the total series and is just significant for duodenal ulcer alone. In the cases with unsatisfactory results there appears to be no significant difference either in sex or in the site of ulcer. We may therefore conclude that the original site of the ulcer, e.g., gastric or duodenal, does not appear to affect the result of gastrectomy. As far as the sex is concerned, male patients appear to do better than female patients to a degree that is just statistically significant.

TABLE VI

RESULTS OF GASTRECTOMY ACCORDING TO AGE

\begin{tabular}{|c|c|c|c|c|c|c|c|c|c|c|c|}
\hline & \multirow[b]{3}{*}{ Age } & \multicolumn{5}{|c|}{ Male } & \multicolumn{5}{|c|}{ Female } \\
\hline & & \multicolumn{3}{|c|}{ Results (Followed Up) } & \multirow{2}{*}{$\begin{array}{c}\text { Dead } \\
\text { and Not } \\
\text { Followed } \\
\text { Up }\end{array}$} & \multirow[b]{2}{*}{ Total } & \multicolumn{3}{|c|}{ Results (Followed Up) } & \multirow{2}{*}{$\begin{array}{c}\text { Dead } \\
\text { and Not } \\
\text { Followed } \\
\text { Up }\end{array}$} & \multirow[b]{2}{*}{ Tota } \\
\hline & & $\begin{array}{l}\text { Groups } \\
1 \text { and } 2\end{array}$ & $\underset{3}{\text { Group }}$ & $\begin{array}{l}\text { Groups } \\
4 \text { and } 5\end{array}$ & & & $\begin{array}{l}\text { Groups } \\
1 \text { and } 2\end{array}$ & $\underset{3}{\text { Group }}$ & $\begin{array}{l}\text { Groups } \\
4 \text { and } 5\end{array}$ & & \\
\hline Gastric ulcer & $\begin{array}{l}\text { Under } 44 \\
45-54 \\
55-64 \\
65+\end{array}$ & $\begin{array}{c}45 \\
(76 \%) \\
52 \% \\
(61 \%) \\
31 \% \\
(64 \%) \\
6 \\
(67 \%)\end{array}$ & $\begin{array}{r}8 \\
22 \\
9 \\
3\end{array}$ & $\begin{array}{c}6 \\
(10.2 \%) \\
9 \\
(10.8 \%) \\
8 \% \\
(17 \%) \\
-\end{array}$ & $\begin{array}{r}4 \\
13 \\
15 \\
9\end{array}$ & $\begin{array}{l}63 \\
96 \\
63 \\
18\end{array}$ & $\begin{array}{c}12 \\
(80 \%) \\
8 \\
(38 \%) \\
15 \% \\
(60 \%) \\
3 \\
(75 \%)\end{array}$ & $\begin{array}{r}- \\
11 \\
8 \\
1\end{array}$ & $\begin{array}{c}33 \\
(20 \%) \\
2 \% \\
(9.5 \%) \\
2 \% \\
(8 \%) \\
-\end{array}$ & $\begin{array}{l}4 \\
1 \\
5 \\
4\end{array}$ & $\begin{array}{r}19 \\
22 \\
30 \\
8\end{array}$ \\
\hline Total & & $\begin{array}{l}134 \\
(67 \%)\end{array}$ & 42 & $\begin{array}{l}23 \\
(12 \%)\end{array}$ & 41 & 240 & $\begin{array}{l}38 \\
(58 \%)\end{array}$ & 20 & $\left(\begin{array}{l}7 \\
(11 \%)\end{array}\right.$ & 14 & 79 \\
\hline Duodenal ulcer & $\begin{array}{l}\text { Under } 44 \\
45-54 \\
55-64 \\
65+\end{array}$ & $\begin{array}{l}119 \\
(68 \%) \\
97 \% \\
(66 \%) \\
50 \\
(74 \%) \\
11 \% \\
(79 \%)\end{array}$ & $\begin{array}{r}33 \\
24 \\
14 \\
3\end{array}$ & $\begin{array}{l}23 \\
(12 \%) \\
25 \% \\
(17 \%) \\
4 \\
(5.8 \%) \\
-\end{array}$ & $\begin{array}{r}16 \\
18 \\
9 \\
5\end{array}$ & $\begin{array}{r}191 \\
164 \\
77 \\
19\end{array}$ & $\begin{array}{l}21 \\
(55 \%) \\
16 \% \\
(59 \%) \\
9 \% \\
(60 \%) \\
(50 \%)\end{array}$ & $\begin{array}{r}12 \\
10 \\
5 \\
-\end{array}$ & $\begin{array}{c}5 \\
(13 \%) \\
1 \% \\
(4 \%) \\
1 \\
(7 \%) \\
1 \% \\
(50 \%)\end{array}$ & $\begin{array}{r}3 \\
1 \\
4 \\
-\end{array}$ & $\begin{array}{r}41 \\
28 \\
19 \\
2\end{array}$ \\
\hline Total & & $\begin{array}{l}277 \\
(69 \%)\end{array}$ & 74 & 52 & 48 & 451 & $\begin{array}{l}47 \\
(57 \%)\end{array}$ & 27 & $\begin{array}{c}8 \\
(9 \cdot 7 \%)\end{array}$ & 8 & 90 \\
\hline
\end{tabular}


TABLE VII

EFFECTS OF SEX AND SITE OF ULCER ON RESULT OF OPERATION

\begin{tabular}{|c|c|c|c|c|c|c|}
\hline & \multicolumn{3}{|c|}{ "Satisfactory" Results } & \multicolumn{3}{|c|}{ “Unsatisfactory” Results } \\
\hline & Male & Female & Total & Male & Female & Total \\
\hline $\begin{array}{l}\text { Gastric ulcer } \\
\text { Duodenal ulcer }\end{array}$ & $\begin{array}{l}\frac{134}{199}=67.3 \% \\
\frac{277}{403}=68.7 \%\end{array}$ & $\begin{array}{l}\frac{38}{65}=58.5 \% \\
\frac{47}{82}=57.3 \%\end{array}$ & $\begin{array}{l}\frac{172}{264}=65 \cdot 15 \% \\
\frac{324}{485}=66.80 \%\end{array}$ & $\begin{array}{r}\frac{23}{199} \\
\frac{52}{403}\end{array}$ & $\begin{array}{r}7 \\
65 \\
8 \\
82\end{array}$ & $\begin{array}{l}\frac{30}{264}=11.4 \% \\
\frac{60}{485}=12.4 \%\end{array}$ \\
\hline
\end{tabular}

(4) Duration of Symptoms before Gastrectomy and Effect on Result of Operation.-It had previously been our impression in the Professorial Unit followup clinic that the longer the duration of symptoms before gastrectomy the better were the results of the operation, particularly in cases of duodenal ulcer. We therefore analysed the results according to the length of symptoms before operation and these figures are represented in Fig. 1.
It will be seen from this graph, however, that there appears to be no statistical trend to support this view and we conclude that the duration of symptoms before operation does not appear to alter the results materially.

(5) Effect of Type of Operation on Result of Gastrectomy.-In the total series the operations were performed by a number of surgeons and no particular scheme of operations was undertaken.

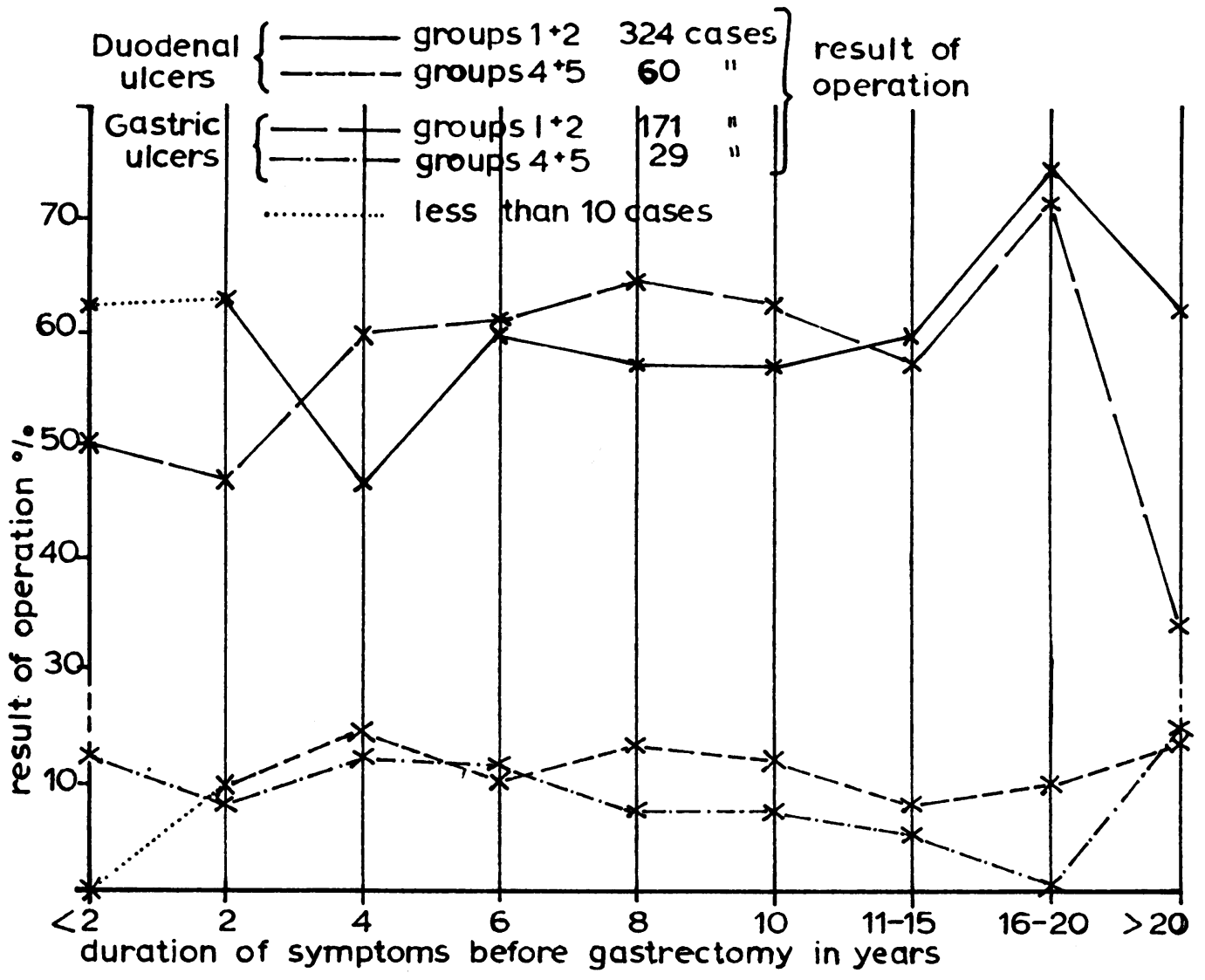

FIG. 1.-Duration of symptoms related to results of gastrectomy. 
Originally the Polya operation was the standard one and 828 gastrectomies of this type were done. The antecolic operation with the afferent loop to the lesser curvature of the stomach was the operation of choice (725 cases) and of these the Hofmeister variation was performed in 235 cases. A limited number of retrocolic operations was performed rather sporadically (103 cases), 39 of which were of the Hofmeister type. Later a number of Billroth 1 gastrectomies were carried out, a total of 127 of this operation being included in this series. The amount of stomach removed varied from $50 \%$ to $90 \%$ throughout all types of operation.

We have carried out analyses to determine if any particular type of operation affected the overall result. We are not assessing only recurrent ulcer rates but all factors influencing the final result of the cases.

The following main comparisons have been made: (i) Polya gastrectomy versus Billroth; (ii) antecolic versus retrocolic Polya; (iii) Polya versus Polya Hofmeister; (iv) a more limited resection of stomach (two-thirds or less) versus a more extensive resection (more than two-thirds). The results are shown in Tables VIII, IX, and X.

After comparing not only percentages but also the total numbers of cases in each group, we have concluded from these figures as follows:-

(a) For gastric ulcer the Billroth operation gives significantly better results than the Polya operation for the total series, and for men alone. In women the difference is not statistically significant but this is probably because the total number of cases is relatively small.

(b) For duodenal ulcer there does not appear to be any significant difference in the results between Polya and Billroth operation.

(c) The extent of gastric resection did not appear to affect significantly the results in either the Billroth or the Polya operations performed for gastric ulcer.

(d) Similarly the amount of stomach removed did not appear to affect the results of Billroth gastrectomy for duodenal ulcer, nor the results of Polya gastrectomy for this condition in the total series. In men alone, however, the more limited resections (two-thirds or less) in the Polya operations for duodenal ulcer were significantly better than the more extensive resections.

(e) The number of cases of retrocolic Polya operation is relatively too small as compared with the antecolic ones to make the percentages of results significant. Only in duodenal ulcers in women does there appear to be a really significant difference and here the antecolic operation appears to be the better one.

$(f)$ The Polya Hofmeister operation does not appear to give better results for duodenal ulcer

TABLE VIII

COMPARISON OF POLYA AND BILLROTH OPERATIONS

\begin{tabular}{|c|c|c|c|c|c|c|c|c|c|c|c|}
\hline \multirow{3}{*}{$\begin{array}{l}\text { Type of } \\
\text { Ulcer }\end{array}$} & \multirow{3}{*}{$\begin{array}{r}\text { Type of } \\
\text { Operation }\end{array}$} & \multirow{3}{*}{$\begin{array}{l}\text { Extent of } \\
\text { Resection }\end{array}$} & \multicolumn{9}{|c|}{ Results } \\
\hline & & & \multicolumn{3}{|c|}{ Male } & \multicolumn{3}{|c|}{ Female } & \multicolumn{3}{|c|}{ Total } \\
\hline & & & $\begin{array}{c}\text { No. } \\
\text { Followed }\end{array}$ & $\begin{array}{l}\text { Groups } \\
1 \text { and } 2\end{array}$ & $\begin{array}{l}\text { Groups } \\
4 \text { and } 5\end{array}$ & $\begin{array}{c}\text { No. } \\
\text { Followed }\end{array}$ & $\begin{array}{l}\text { Groups } \\
1 \text { and } 2\end{array}$ & $\begin{array}{l}\text { Groups } \\
4 \text { and } 5\end{array}$ & $\begin{array}{c}\text { No. } \\
\text { Followed }\end{array}$ & $\begin{array}{l}\text { Groups } \\
1 \text { and } 2\end{array}$ & $\begin{array}{l}\text { Groups } \\
4 \text { and } 5\end{array}$ \\
\hline Gastric & $\begin{array}{l}\text { Polya } \\
\text { Billroth } \\
\text { Total (Polya and Bilroth) }\end{array}$ & $\begin{array}{l}\text { Two-thirds or less } \\
\text { Over two-thirds } \\
\text { Total of all cases } \\
\text { Two-thirds or less } \\
\text { Over two-thirds } \\
\text { Total of all cases } \\
\text { Two-thirds or less } \\
\text { Over two-thirds } \\
\text { Total of all cases }\end{array}$ & $\begin{array}{r}99 \\
55 \\
154 \\
40 \\
5 \\
45 \\
139 \\
60 \\
199\end{array}$ & \begin{tabular}{|c}
$63(63 \cdot 6)$ \\
$30(54 \cdot 5)$ \\
$93(60 \cdot 4)$ \\
$37(92 \cdot 5)$ \\
4 \\
$41(91 \cdot 1)$ \\
$100(71 \cdot 9)$ \\
$34(56 \cdot 7)$ \\
$134(67 \cdot 3)$
\end{tabular} & $\begin{array}{r}5 \\
17 \\
22 \\
1 \\
0 \\
1 \\
6 \\
17 \\
23\end{array}$ & $\begin{array}{r}22 \\
22 \\
44 \\
16 \\
5 \\
21 \\
38 \\
27 \\
65\end{array}$ & $\begin{array}{r}14(63 \cdot 6) \\
9(40.9) \\
23(52 \cdot 3) \\
11(68 \cdot 7) \\
33(14) \\
14(66 \cdot 7) \\
25(65 \cdot 8) \\
12(44 \cdot 4) \\
37(56 \cdot 9)\end{array}$ & $\begin{array}{l}3 \\
4 \\
7 \\
7 \\
0 \\
0 \\
0 \\
3 \\
4 \\
\mathbf{7}\end{array}$ & $\begin{array}{r}121 \\
77 \\
198 \\
56 \\
10 \\
66 \\
177 \\
87 \\
264\end{array}$ & $\begin{array}{r}77(63 \cdot 6) \\
39(50 \cdot 6) \\
116(58.6) \\
48(85 \cdot 7) \\
7(70 \cdot 0) \\
55(83 \cdot 3) \\
125(78 \cdot 5) \\
46(52.9) \\
171(64 \cdot 8)\end{array}$ & $\begin{array}{r}8 \\
21 \\
29 \\
1 \\
0 \\
1 \\
9 \\
21 \\
30\end{array}$ \\
\hline Duodenal & $\begin{array}{l}\text { Polya } \\
\text { Billroth } \\
\text { Total (Polya and Billroth) }\end{array}$ & $\begin{array}{l}\text { Two-thirds or less } \\
\text { Over two-thirds } \\
\text { Total of all cases } \\
\text { Two-thirds or less } \\
\text { Over two-thirds } \\
\text { Total of all cases } \\
\text { Two-thirds or less } \\
\text { Over two-thirds } \\
\text { Total of all cases }\end{array}$ & $\begin{array}{r}277 \\
92 \\
369 \\
26 \\
8 \\
34 \\
303 \\
100 \\
403\end{array}$ & $\begin{array}{r}197(71 \cdot 1)) \\
56(60 \cdot 9) \\
253(68 \cdot 6) \\
17(65 \cdot 4) \\
7(87 \cdot 5) \\
24(70 \cdot 6) \\
214(70 \cdot 6) \\
63(63 \cdot 0) \\
277(68 \cdot 7)\end{array}$ & $\begin{array}{r}32 \\
16 \\
48 \\
4 \\
0 \\
4 \\
36 \\
16 \\
52\end{array}$ & $\begin{array}{r}61 \\
15 \\
76 \\
6 \\
0 \\
6 \\
67 \\
15 \\
82\end{array}$ & $\begin{array}{r}35(57 \cdot 4) \\
8(53 \cdot 3) \\
43(56 \cdot 6) \\
4 \\
0 \\
4 \\
39(58 \cdot 2) \\
8(53 \cdot 3) \\
47(57 \cdot 3)\end{array}$ & $\begin{array}{l}5 \\
3 \\
8 \\
0 \\
0 \\
0 \\
5 \\
3 \\
8\end{array}$ & $\begin{array}{r}338 \\
107 \\
445 \\
32 \\
8 \\
40 \\
370 \\
115 \\
485\end{array}$ & $\begin{array}{r}232(68 \cdot 6) \\
64(59 \cdot 8) \\
296(66 \cdot 5) \\
21(65 \cdot 6) \\
7(87 \cdot 5) \\
28(70 \cdot 0) \\
253(68 \cdot 4) \\
71(61 \cdot 7) \\
324(66 \cdot 8)\end{array}$ & $\begin{array}{r}37 \\
19 \\
56 \\
4 \\
0 \\
4 \\
41 \\
19 \\
60\end{array}$ \\
\hline Total & $\begin{array}{l}\text { Polya } \\
\text { Billroth } \\
\text { Total (Polya and Billroth) }\end{array}$ & $\begin{array}{l}\text { Two-thirds or less } \\
\text { Over two-thirds } \\
\text { Total of all cases } \\
\text { Two-thirds or less } \\
\text { Over two-thirds } \\
\text { Total of all cases } \\
\text { Two-thirds or less } \\
\text { Over two-thirds } \\
\text { Total of all cases }\end{array}$ & $\begin{array}{r}376 \\
147 \\
523 \\
66 \\
13 \\
79 \\
442 \\
160 \\
602\end{array}$ & $\begin{array}{r}260(69 \cdot 1) \\
86(58 \cdot 5) \\
346(66 \cdot 2) \\
54(81 \cdot 8) \\
11(84 \cdot 6) \\
65(82 \cdot 3) \\
314(71 \cdot 0) \\
97(60 \cdot 6) \\
411(68 \cdot 3)\end{array}$ & $\begin{array}{r}37 \\
33 \\
70 \\
5 \\
0 \\
5 \\
42 \\
33 \\
75\end{array}$ & $\begin{array}{r}83 \\
37 \\
120 \\
22 \\
5 \\
27 \\
105 \\
42 \\
147\end{array}$ & $\begin{array}{l}49(59 \cdot 0) \\
17(45.9) \\
66(55.0) \\
15(68 \cdot 2) \\
3(60.0) \\
18(66 \cdot 7) \\
64(61 \cdot 0) \\
20(47.6) \\
84(57 \cdot 1)\end{array}$ & $\begin{array}{r}8 \\
7 \\
15 \\
0 \\
0 \\
0 \\
8 \\
7 \\
15\end{array}$ & \begin{tabular}{r|}
459 \\
184 \\
643 \\
88 \\
18 \\
106 \\
547 \\
202 \\
749
\end{tabular} & $\begin{array}{r}309(67 \cdot 3) \\
103(56 \cdot 0) \\
412(64 \cdot 1) \\
69(78 \cdot 4) \\
14(77 \cdot 8) \\
83(78 \cdot 3) \\
378(69 \cdot 1) \\
117(57.9) \\
495(66 \cdot 1)\end{array}$ & $\begin{array}{r}45 \\
40 \\
85 \\
5 \\
0 \\
5 \\
50 \\
40 \\
90\end{array}$ \\
\hline
\end{tabular}

Percentages of groups 1 and 2 results are shown in parentheses. 
TABLE IX

COMPARISON OF ANTECOLIC AND RETROCOLIC POLYA OPERATIONS

\begin{tabular}{|c|c|c|c|c|c|c|c|c|c|c|c|}
\hline \multirow{3}{*}{$\begin{array}{l}\text { Type of } \\
\text { Ulcer }\end{array}$} & \multirow{3}{*}{$\begin{array}{r}\text { Type of } \\
\text { Operation }\end{array}$} & \multirow{3}{*}{$\begin{array}{l}\text { Extent of } \\
\text { Resection }\end{array}$} & \multicolumn{9}{|c|}{ Results } \\
\hline & & & \multicolumn{3}{|c|}{ Male } & \multicolumn{3}{|c|}{ Female } & \multicolumn{3}{|c|}{ Total } \\
\hline & & & $\begin{array}{c}\text { No. } \\
\text { Followed }\end{array}$ & $\begin{array}{l}\text { Groups } \\
1 \text { and } 2\end{array}$ & $\begin{array}{l}\text { Groups } \\
4 \text { and } 5\end{array}$ & $\begin{array}{c}\text { No. } \\
\text { Followed }\end{array}$ & $\begin{array}{l}\text { Groups } \\
1 \text { and } 2\end{array}$ & $\begin{array}{l}\text { Groups } \\
4 \text { and } 5\end{array}$ & $\begin{array}{c}\text { No. } \\
\text { Followed }\end{array}$ & $\begin{array}{l}\text { Groups } \\
1 \text { and } 2\end{array}$ & $\begin{array}{l}\text { Groups } \\
4 \text { and } 5\end{array}$ \\
\hline Gastric & $\begin{array}{l}\text { Antecolic Polya } \\
\text { Retrocolic Polya }\end{array}$ & $\begin{array}{l}\text { Two-thirds or less } \\
\text { Over two-thirds } \\
\text { Total of all cases } \\
\text { Two-thirds or less } \\
\text { Over two-thirds } \\
\text { Total of all cases } \\
\end{array}$ & $\begin{array}{r}87 \\
48 \\
135 \\
12 \\
7 \\
19\end{array}$ & $\begin{array}{l}54(62 \cdot 1) \\
24(50 \cdot 0) \\
78(57 \cdot 8) \\
9(75 \cdot 0) \\
6 \\
15(78.9)\end{array}$ & $\begin{array}{r}5 \\
16 \\
21 \\
0 \\
1 \\
1\end{array}$ & $\begin{array}{r}20 \\
17 \\
37 \\
2 \\
5 \\
7\end{array}$ & $\begin{array}{r}12(60 \cdot 0) \\
7(41 \cdot 2) \\
19(51 \cdot 4) \\
2 \\
2 \\
4\end{array}$ & $\begin{array}{l}3 \\
3 \\
6 \\
0 \\
1 \\
1\end{array}$ & $\begin{array}{r}107 \\
65 \\
172 \\
14 \\
12 \\
26\end{array}$ & $\begin{array}{r}66(61 \cdot 7) \\
31(47 \cdot 5) \\
97(56 \cdot 4) \\
11(78 \cdot 6) \\
8(66 \cdot 7) \\
19(73 \cdot 1)\end{array}$ & $\begin{array}{r}8 \\
19 \\
27 \\
0 \\
2 \\
2\end{array}$ \\
\hline Duodenal & $\begin{array}{l}\text { Antecolic Polya } \\
\text { Retrocolic Polya }\end{array}$ & $\begin{array}{l}\text { Two-thirds or less } \\
\text { Over two-thirds } \\
\text { Total of all cases } \\
\text { Two-thirds or less } \\
\text { Over two-thirds } \\
\text { Total of all cases } \\
\end{array}$ & $\begin{array}{r}245 \\
77 \\
322 \\
32 \\
15 \\
47\end{array}$ & $\begin{array}{r}175(71 \cdot 4) \\
45(58 \cdot 4) \\
220(68 \cdot 3) \\
22(68 \cdot 8) \\
11(73 \cdot 3) \\
33(70 \cdot 2)\end{array}$ & $\begin{array}{r}29 \\
14 \\
43 \\
3 \\
2 \\
2 \\
5\end{array}$ & $\begin{array}{r}54 \\
13 \\
67 \\
7 \\
2 \\
9\end{array}$ & $\begin{array}{l}32 \\
8(61 \cdot 5) \\
40(59 \cdot 7) \\
3(42 \cdot 9) \\
0 \\
3(33 \cdot 3)\end{array}$ & $\begin{array}{l}5 \\
2 \\
7 \\
0 \\
1 \\
1\end{array}$ & $\begin{array}{r}299 \\
90 \\
389 \\
39 \\
17 \\
56\end{array}$ & \begin{tabular}{|r|}
$207(69 \cdot 2)$ \\
$53(58 \cdot 9)$ \\
$260(66 \cdot 8)$ \\
$25(64 \cdot 1)$ \\
$11(64 \cdot 7)$ \\
$36(64 \cdot 3)$
\end{tabular} & $\begin{array}{r}34 \\
16 \\
50 \\
3 \\
3 \\
6\end{array}$ \\
\hline Total & $\begin{array}{l}\text { Antecolic Polya } \\
\text { Retrocolic Polya }\end{array}$ & $\begin{array}{l}\text { Two-thirds or less } \\
\text { Over two-thirds } \\
\text { Total of all cases } \\
\text { Two-thirds or less } \\
\text { Over two-thirds } \\
\text { Total of all cases }\end{array}$ & $\begin{array}{r}332 \\
125 \\
457 \\
44 \\
22 \\
66\end{array}$ & $\begin{array}{r}229(69 \cdot 0) \\
69(55 \cdot 2) \\
298(65 \cdot 2) \\
31(70 \cdot 5) \\
17(77 \cdot 3) \\
48(72 \cdot 7)\end{array}$ & $\begin{array}{r}34 \\
30 \\
64 \\
3 \\
3 \\
6\end{array}$ & $\begin{array}{r}74 \\
30 \\
104 \\
9 \\
7 \\
16\end{array}$ & $\begin{array}{l}44(59 \cdot 5) \\
15(50 \cdot 0) \\
59(56 \cdot 7) \\
5 \\
2 \\
7\end{array}$ & $\begin{array}{r}8 \\
5 \\
13 \\
0 \\
2 \\
2\end{array}$ & $\begin{array}{r}406 \\
155 \\
561 \\
53 \\
29 \\
82\end{array}$ & $\begin{array}{r}273(67 \cdot 2) \\
84(54 \cdot 2) \\
357(63 \cdot 6) \\
36(67 \cdot 9) \\
19(65 \cdot 5) \\
55(67 \cdot 1)\end{array}$ & $\begin{array}{r}42 \\
35 \\
77 \\
3 \\
5 \\
8\end{array}$ \\
\hline
\end{tabular}

Percentages of groups 1 and 2 results are shown in parentheses. Totals of Polya operations are given in Table VIII.

TABLE X

COMPARISON OF POLYA AND POLYA HOFMEISTER OPERATIONS

\begin{tabular}{|c|c|c|c|c|c|c|c|c|c|c|c|}
\hline \multirow{3}{*}{$\begin{array}{l}\text { Type of } \\
\text { Ulcer }\end{array}$} & \multirow{3}{*}{$\begin{array}{c}\text { Type of } \\
\text { Operation }\end{array}$} & \multirow{3}{*}{$\begin{array}{l}\text { Extent of } \\
\text { Resection }\end{array}$} & \multicolumn{9}{|c|}{ Results } \\
\hline & & & \multicolumn{3}{|c|}{ Male } & \multicolumn{3}{|c|}{ Female } & \multicolumn{3}{|c|}{ Totai } \\
\hline & & & $\begin{array}{c}\text { No. } \\
\text { Followed }\end{array}$ & $\begin{array}{l}\text { Groups } \\
1 \text { and } 2 \\
\end{array}$ & $\begin{array}{l}\text { Groups } \\
4 \text { and } 5 \\
\end{array}$ & $\begin{array}{c}\text { No. } \\
\text { Followed } \\
\end{array}$ & $\begin{array}{l}\text { Groups } \\
1 \text { and } 2\end{array}$ & $\begin{array}{l}\text { Groups } \\
4 \text { and } 5\end{array}$ & $\begin{array}{c}\text { No. } \\
\text { Followed }\end{array}$ & $\begin{array}{l}\text { Groups } \\
1 \text { and } 2\end{array}$ & $\begin{array}{l}\text { Groups } \\
4 \text { and } 5 \\
\end{array}$ \\
\hline Gastric & $\begin{array}{l}\text { Polya } \\
\text { Polya Hofmeister }\end{array}$ & $\begin{array}{l}\text { Two-thirds or less } \\
\text { Over two-thirds } \\
\text { Total of all cases } \\
\text { Two-thirds or less } \\
\text { Over two-thirds } \\
\text { Total of all cases }\end{array}$ & $\begin{array}{r}71 \\
40 \\
111 \\
28 \\
15 \\
43\end{array}$ & $\begin{array}{l}46(64 \cdot 8) \\
22(55 \cdot 0) \\
68(61 \cdot 3) \\
17(60 \cdot 7) \\
8(53 \cdot 3) \\
25(58 \cdot 1)\end{array}$ & $\begin{array}{r}4 \\
11 \\
15 \\
1 \\
6 \\
7\end{array}$ & $\begin{array}{r}11 \\
19 \\
30 \\
11 \\
3 \\
14\end{array}$ & $\begin{array}{r}4(36 \cdot 4) \\
7(36 \cdot 8) \\
11(36 \cdot 7) \\
10(90 \cdot 9) \\
2(25 \cdot 7) \\
12(85 \cdot 7)\end{array}$ & $\begin{array}{l}3 \\
3 \\
6 \\
0 \\
1 \\
1\end{array}$ & $\begin{array}{r}82 \\
59 \\
141 \\
39 \\
18 \\
57\end{array}$ & $\begin{array}{l}50(61 \cdot 0) \\
29(49 \cdot 2) \\
79(56 \cdot 0) \\
27(69 \cdot 2) \\
10(55 \cdot 5) \\
37(64 \cdot 9)\end{array}$ & $\begin{array}{r}7 \\
14 \\
21 \\
1 \\
7 \\
8\end{array}$ \\
\hline Duodenal & $\begin{array}{l}\text { Polya } \\
\text { Polya Hofmeister }\end{array}$ & $\begin{array}{l}\text { Two-thirds or less } \\
\text { Over two-thirds } \\
\text { Total of all cases } \\
\text { Two-thirds or less } \\
\text { Over two-thirds } \\
\text { Total of all cases } \\
\end{array}$ & $\begin{array}{r}174 \\
63 \\
237 \\
103 \\
29 \\
132 \\
\end{array}$ & $\begin{array}{r}123(70 \cdot 7) \\
39(61 \cdot 9) \\
162(68 \cdot 4) \\
74(71 \cdot 8) \\
17(58 \cdot 6) \\
91(68 \cdot 9) \\
\end{array}$ & $\begin{array}{r}18 \\
9 \\
27 \\
14 \\
7 \\
21 \\
\end{array}$ & $\begin{array}{r}43 \\
12 \\
55 \\
18 \\
3 \\
21 \\
\end{array}$ & 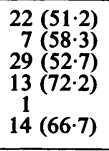 & $\begin{array}{l}4 \\
3 \\
7 \\
1 \\
0 \\
1\end{array}$ & $\begin{array}{r}217 \\
75 \\
292 \\
121 \\
32 \\
153 \\
\end{array}$ & $\begin{array}{r}145(66 \cdot 8) \\
46(61 \cdot 3) \\
191(65 \cdot 4) \\
87(71 \cdot 9) \\
18(56 \cdot 3) \\
105(68 \cdot 6) \\
\end{array}$ & $\begin{array}{r}22 \\
12 \\
34 \\
15 \\
7 \\
22 \\
\end{array}$ \\
\hline Total & $\begin{array}{l}\text { Polya } \\
\text { Polya Hofmeister }\end{array}$ & $\begin{array}{l}\text { Two-thirds or less } \\
\text { Over two-thirds } \\
\text { Total of all cases } \\
\text { Two-thirds or less } \\
\text { Over two-thirds } \\
\text { Total of all cases }\end{array}$ & $\begin{array}{r}245 \\
103 \\
348 \\
131 \\
44 \\
175\end{array}$ & $\begin{array}{r}169(69 \cdot 0) \\
61(59 \cdot 2) \\
230(66 \cdot 1) \\
91(69 \cdot 5) \\
25(56 \cdot 8) \\
116(66 \cdot 3)\end{array}$ & $\begin{array}{l}22 \\
20 \\
42 \\
15 \\
13 \\
28\end{array}$ & $\begin{array}{r}54 \\
31 \\
85 \\
29 \\
6 \\
35\end{array}$ & $\begin{array}{l}26(48 \cdot 1) \\
14(45 \cdot 2) \\
40(47 \cdot 1) \\
23(79 \cdot 3) \\
3 \\
26(74 \cdot 3)\end{array}$ & $\begin{array}{r}7 \\
6 \\
13 \\
1 \\
1 \\
2\end{array}$ & $\begin{array}{r}299 \\
134 \\
433 \\
160 \\
50 \\
210\end{array}$ & $\begin{array}{r}195(65 \cdot 2) \\
75(56 \cdot 0) \\
270(62 \cdot 4) \\
114(71 \cdot 3) \\
28(56 \cdot 0) \\
142(67 \cdot 6)\end{array}$ & $\begin{array}{l}29 \\
26 \\
55 \\
16 \\
14 \\
30\end{array}$ \\
\hline
\end{tabular}

Percentages of groups 1 and 2 results are shown in parentheses. Totals of Polya operations are given in Table VIII.

than the original Polya operation and there is similarly no difference in the total number done for gastric ulcer. For gastric ulcer in women alone, however, the Polya Hofmeister operation is significantly better than the Polya operation.

(6) Indications for Gastrectomy and their Effect on Results.-From the notes made at the time of the original operation we have classified the indications for the operation. We considered 10 primary indications for gastrectomy but in some cases two or more of these indications may have been present. In Table XI we show an analysis of the results of operations for duodenal ulcer and gastric ulcer in comparison with nine primary indications. (The tenth indication was stomal ulcer after previous gastroenterostomy and this, of course, does not apply in these two conditions.) Where two or more indications have been present we have taken the more serious one as the primary one. In addition to its interest as an analysis of the indications for gastrectomy, the table shows that results of gastrectomy do not appear to be related in any significant way to the indication for the operation.

Of the 955 patients in the total series, 360 had suffered a complication of their ulcer, such as stenosis, bleeding, perforation, etc. In the 
TABLE XI

INDICATIONS FOR GASTRECTOMY AND THEIR EFFECT ON RESULT OF OPERATION

\begin{tabular}{|c|c|c|c|c|c|c|c|c|c|}
\hline & \multirow[b]{2}{*}{$\begin{array}{l}\text { Priniary Indication for } \\
\text { Gastrectomy }\end{array}$} & \multicolumn{4}{|c|}{ Duodenal Ulcers - Results } & \multicolumn{4}{|c|}{ Gastric Ulcers-Results } \\
\hline & & $\begin{array}{l}\text { Groups } \\
1 \text { and } 2\end{array}$ & $\underset{3}{\text { Groups }}$ & $\begin{array}{l}\text { Groups } \\
4 \text { and } 5\end{array}$ & $\begin{array}{c}\text { Total } \\
\text { Followed } \\
\text { Up }\end{array}$ & $\begin{array}{l}\text { Groups } \\
1 \text { and } 2\end{array}$ & $\underset{3}{\text { Group }}$ & $\begin{array}{l}\text { Groups } \\
4 \text { and } 5\end{array}$ & $\begin{array}{l}\text { Total } \\
\text { Followed } \\
\text { Up }\end{array}$ \\
\hline Male & $\begin{array}{l}\text { Pain (indigestion etc.) } \\
\text { Pain, etce, plus previous perforation } \\
\text { Repeated previous haemorrhages } \\
\text { Haemorrhage at time of operation } \\
\text { (acute ulcer) time of operation } \\
\text { Haemorrhage at time } \\
\text { (chronic ulcer) } \\
\text { Pyloric stenosis } \\
\text { Hour-glass obstruction } \\
\text { Fear of malignancy } \\
\begin{array}{l}\text { Original ulcer still active plus gastro- } \\
\text { enterostomy }\end{array}\end{array}$ & $\begin{array}{l}176 \\
(67 \cdot 2) \\
24 \\
(72 \cdot 7) \\
25 \\
(78 \cdot 1) \\
- \\
14 \\
(73 \cdot 7) \\
38 \\
(73 \cdot 1) \\
= \\
- \\
-\end{array}$ & $\begin{array}{c}52 \\
(19 \cdot 8) \\
6 \\
(18 \cdot 2) \\
3 \\
(9 \cdot 4) \\
- \\
3 \\
(15 \cdot 8) \\
8 \\
(15 \cdot 4) \\
= \\
- \\
2\end{array}$ & $\begin{array}{c}34 \\
(13) \\
3 \\
(9 \cdot 1) \\
4 \\
(12 \cdot 5) \\
1 \\
2 \\
(10 \cdot 5) \\
6 \\
(11 \cdot 5) \\
= \\
- \\
2\end{array}$ & $\begin{array}{r}262 \\
33 \\
32 \\
1 \\
19 \\
52 \\
= \\
- \\
4\end{array}$ & $\begin{array}{c}88 \\
(65 \cdot 7) \\
6 \\
(100) \\
5 \\
(62 \cdot 5) \\
1 \\
10 \\
(71 \cdot 4) \\
9 \\
(81 \cdot 8) \\
1 \\
14 \\
(63 \cdot 6) \\
-\end{array}$ & $\begin{array}{c}30 \\
(22 \cdot 4) \\
- \\
3 \\
(27 \cdot 5) \\
1 \\
2 \\
(14 \cdot 3) \\
1 \\
(9 \cdot 1) \\
-5 \\
(22 \cdot 7) \\
-\end{array}$ & $\begin{array}{c}16 \\
(11) \\
- \\
- \\
1 \\
2 \\
(14 \cdot 3) \\
1 \\
(9 \cdot 1) \\
-3 \\
(13 \cdot 6) \\
-\end{array}$ & $\begin{array}{r}134 \\
6 \\
8 \\
3 \\
14 \\
11 \\
1 \\
22 \\
-\end{array}$ \\
\hline Female & $\begin{array}{l}\text { Pain (indigestion, etc.) } \\
\text { Pain, etc., plus previous perforation } \\
\text { Repeated previous haemorrhages } \\
\text { Haemorrhage at time of operation } \\
\text { (acute ulcer) } \\
\text { Haemorrhage at time of operation } \\
\text { (chronic ulcer) } \\
\text { Pyloric stenosis } \\
\text { Hour-glass obstruction } \\
\text { Fear of malignancy } \\
\text { Original ulcer still active plus gastro- } \\
\text { enterostomy }\end{array}$ & $\begin{array}{l}25 \\
(50) \\
3 \\
(75) \\
= \\
2 \\
17 \\
(81) \\
= \\
-\end{array}$ & $\begin{array}{r}19 \\
(38) \\
- \\
- \\
1 \\
4 \\
\frac{(19)}{2} \\
-\end{array}$ & $\begin{array}{l}6 \\
- \\
2 \\
- \\
- \\
- \\
= \\
=\end{array}$ & $\begin{array}{r}50 \\
4 \\
2 \\
- \\
3 \\
21 \\
-2 \\
-\end{array}$ & $\begin{array}{c}22 \\
(53 \cdot 7) \\
1 \\
1 \\
1 \\
3 \\
5 \\
1 \\
4 \\
-\end{array}$ & $\begin{array}{c}13 \\
(31 \cdot 7) \\
1 \\
1 \\
1 \\
- \\
1 \\
- \\
\end{array}$ & $\begin{array}{c}6 \\
(14 \cdot 6) \\
- \\
= \\
1 \\
- \\
= \\
=\end{array}$ & $\begin{array}{r}41 \\
2 \\
2 \\
1 \\
5 \\
5 \\
2 \\
7 \\
\end{array}$ \\
\hline
\end{tabular}

Actual number of cases followed up in each group is given. Percentages are given in parentheses except where number of cases is very small.

772 men there were 287 such cases $(37 \cdot 1 \%)$ and in the 183 women 73 cases $(39 \cdot 8 \%)$. Altogether $11 \cdot 8 \%$ of the total had pyloric stenosis, $8.6 \%$ had previously had a perforation, $7.7 \%$ had had previous repeated haemorrhages, and a further $6 \%$ were operated on as emergencies for haemorrhage.

\section{OTHER FACTORS INFLUENCED BY GASTRECTOMY}

It has been pointed out that the "result" discussed so far is the overall result of operation. The influence of gastrectomy on some other factors will now be analysed.

EARNING Capacity afTer Gastrectomy.-We have compared the earning capacity of each patient before and after operation as far as possible. Earning capacity rather than actual earning was taken, since in quite a number of cases wages or salaries have been affected by other factors unconnected with gastrectomy. In such instances, although the actual earnings have changed, we have specified earning capacity as "no change". In some patients, particularly women, there was no paying occupation before or after operation and these have been excluded, so that the total number available for assessment in this group is 749 . We have taken all ulcer, age, and sex groups together and have classified them according to the result of gastrectomy. Of the good results (groups 1 and 2), $39.3 \%$ showed an increase in earning capacity (11\% having as much as a $50 \%$ increase), while only $3.2 \%$ showed a decrease. In the poor results (groups 4 and 5), 12.2\% had an increased earning capacity while $37.8 \%$ showed a decrease and this deterioration was adjudged to be due to the operation result. Group 3 results, which had been assessed as not interfering with normal life, showed a $27.6 \%$ increase and only an $8 \%$ decrease in earning capacity which is considered to be a fair justification of the method of assessment.

AMOUNT OF TIME LOST FROM WORK AFTER GASTRECTOMY.-The same general principles as above were used to assess working time lost per year before and after gastrectomy. Time lost was only considered in relation to gastrectomy, and where loss was quite obviously not due to result of the operation the case has either been excluded or a corrected estimate has been given. The number of patients available for analysis is again 749 , the same cases as used in the evaluation of earning capacity. The cases are again divided according to the results of gastrectomy (Table XII). It will be seen that there is a significant drop in the amount of time lost 
TABLE XII

WORKING TIME LOST BEFORE AND AFTER GASTRECTOMY

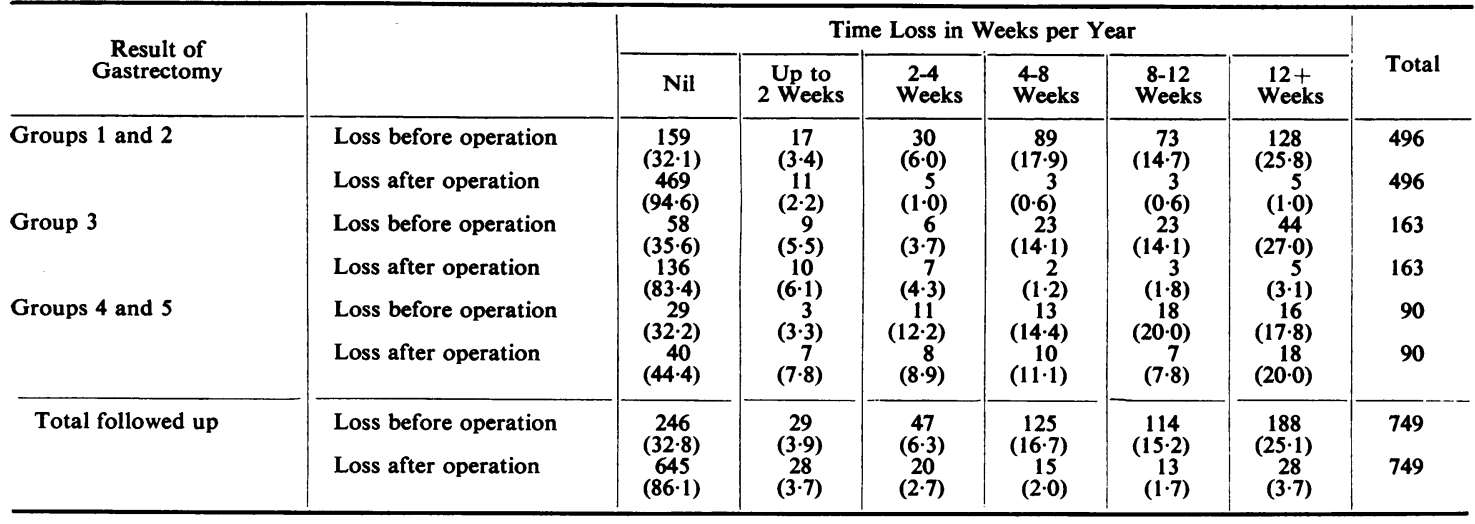

Percentages are shown in parentheses.

from work when the patient has a good result from the operation (groups 1 and 2), and that the same applies in the patients who only had a moderate result (group 3). The amount of time lost by patients in groups 4 and 5 was not greatly altered. The fall of earning capacity in these patients allied to no increase of time loss was usually due to change of occupation.

OCCUPATION BEFORE AND AFTER GASTRECTOMY.Occupations were classified according to the five social status groups specified by the General Register Office. Changes after operation would therefore only become apparent if the patient moved from one broad social group to another. In groups 1 and 2, $88.9 \%$ and in group $3,82.8 \%$ showed no change. In groups 4 and $5,71 \cdot 1 \%$ remained unchanged but $22 \cdot 2 \%$ had moved down the occupation scale.

Gastro-duodenal Haemorrhage and Anaemia. - The occurrence of gastrointestinal haemorrhage as shown by haematemesis, malaena, or the evidence of anaemia before operation in the 955 cases operated upon and after operation in the 826 cases followed up is shown in Table XIII.

The actual haemoglobin levels of patients at the time of admission for operation are shown in Table XIV. Some of these were admitted as emergencies with bleeding or came in with anaemia and would have been transfused before operation was actually undertaken. We estimated the haemoglobin levels in 771 of the 826 cases followed up and the results of these are also shown in Table XIV.

TABLE XIII

GASTROINTESTINAL HAEMORRHAGE BEFORE AND AFTER OPERATION

\begin{tabular}{l|c|c|c|c}
\hline & $\begin{array}{c}\text { No } \\
\text { Haemorrhage }\end{array}$ & $\begin{array}{c}\text { Haematemesis with } \\
\text { or without } \\
\text { Melaena }\end{array}$ & Melaena & Total \\
\hline $\begin{array}{l}\text { Before operation } \\
\text { After operation }\end{array}$ & $\begin{array}{c}694(72 \cdot 7) \\
774(93 \cdot 7)\end{array}$ & $\begin{array}{c}172(18 \cdot 0) \\
6(0 \cdot 7)\end{array}$ & $\begin{array}{r}65(6 \cdot 8) \\
4(0 \cdot 5)\end{array}$ & $\begin{array}{c}95(2 \cdot 5) \\
42(5 \cdot 1)\end{array}$ \\
\hline
\end{tabular}

Percentages are shown in parentheses.

"The cases shown as "anaemia after operation" had needed treatment, and the anaemia had developed after the immediate period of the operation.

TABLE XIV

HAEMOGLOBIN LEVELS BEFORE AND AFTER OPERATION

\begin{tabular}{|c|c|c|c|c|c|c|c|c|c|c|c|}
\hline & & \multicolumn{7}{|c|}{ Levels of Haemoglobin as $\%$ of Normal } & \multirow{2}{*}{$\begin{array}{c}\text { No. } 4 \text { with } \\
\text { Known } \\
\text { Haemoglobin }\end{array}$} & \multirow{2}{*}{$\begin{array}{c}\text { Not } \\
\text { Known } \\
\text { Haemoglobin }\end{array}$} & \multirow{2}{*}{ Total } \\
\hline & & $\overline{\text { Mean Level }}$ & $90+$ & $\overline{80-89}$ & $70-79$ & $60-69$ & $50-59$ & Under 50 & & & \\
\hline Pre-operative & $\begin{array}{l}\text { Male } \\
\text { Female }\end{array}$ & $\begin{array}{r}102 \cdot 8 \\
92 \cdot 5\end{array}$ & $\begin{array}{l}88 \cdot 0 \\
66 \cdot 3\end{array}$ & $\begin{array}{r}4 \cdot 4 \\
18 \cdot 5\end{array}$ & $\begin{array}{l}2 \cdot 8 \\
7.9\end{array}$ & $\begin{array}{l}1.7 \\
2.8\end{array}$ & $\begin{array}{l}1.1 \\
0.6\end{array}$ & $\begin{array}{l}2.0 \\
3.9\end{array}$ & $\begin{array}{l}752 \\
178\end{array}$ & $\begin{array}{r}20 \\
5\end{array}$ & $\begin{array}{l}772 \\
183\end{array}$ \\
\hline Post-operative & $\begin{array}{l}\text { Male } \\
\text { Female }\end{array}$ & $\begin{array}{l}94 \cdot 3 \\
86 \cdot 6\end{array}$ & $\begin{array}{l}71.0 \\
49.6\end{array}$ & $\begin{array}{l}16 \cdot 5 \\
22.5\end{array}$ & $\begin{array}{r}7 \cdot 8 \\
14 \cdot 3\end{array}$ & $\begin{array}{l}3 \cdot 5 \\
6 \cdot 1\end{array}$ & $\begin{array}{l}1 \cdot 0 \\
5 \cdot 5\end{array}$ & $\begin{array}{l}0.2 \\
2 \cdot 0\end{array}$ & $\begin{array}{l}624 \\
147\end{array}$ & $\begin{array}{l}42 \\
13\end{array}$ & $\begin{array}{l}666 \\
160\end{array}$ \\
\hline
\end{tabular}

*The number of cases where the haemoglobin level was known is given for men and women before and after operation. The figures given at each haemoglobin level are percentages of those totals. 
The figures show a significant fall in the mean level of haemoglobin in both sexes after operation. Although the mean level in women is lower than in men both before and after operation, in this series the actual fall in men $(8.5 \%)$ was greater than in women $(5.9 \%)$.

BODY Weight Before AND AFTER GASTRECTOMY. -In all cases where we have sufficient information we have calculated the patient's actual body weight as a percentage above or below their standard body weight for age, height, and sex. The standard weights used are those given in the tables of Kemsley (1952). In Table XV we show these results for duodenal ulcer and for gastric ulcer cases in both sexes. To simplify the table we have expressed the number of cases in each instance as a percentage of the total, the latter being given in each group at the bottom of the table section. For instance, in the "best ever" weights for male duodenal ulcer cases, $42 \%$ of the total of 264 were at standard weight and this represents 111 patients. Each patient's weight had been noted on a number of occasions and these are interpreted as follows:- (a) "Best Ever" Weight.-This is the highest the patient can remember attaining at any time before operation.

(b) Weight just before Operation.-This is the weight recorded in the patient's notes in hospital immediately before operation. (Since patients weighed at follow-up examinations were fully dressed, the weights have been suitably adjusted to be comparable.)

(c) Weight One Year after Operation.-These weights were available in most instances from the notes made at the routine gastrectomy follow-up clinic.

(d) Weight at Time of Follow-up.-These were the weights that we actually recorded in our investigation. The weights in this group include those of all cases however long the time since operation. Thus cases operated on as long ago as six years are included but also patients operated on only one year ago would also be represented.

We have taken the mean weight in duodenal ulcer and in gastric ulcer cases for both sexes and for both

TABLE XV

RESULT OF OPERATION AND WEIGHTS AT DIFFERENT TIMES

\begin{tabular}{|c|c|c|c|c|c|c|c|c|c|c|c|c|c|c|c|c|c|}
\hline \multirow{3}{*}{ Group } & \multirow{3}{*}{$\begin{array}{c}\text { Result } \\
\text { of Operation }\end{array}$} & \multicolumn{4}{|c|}{ Best Ever Weight } & \multicolumn{4}{|c|}{ Weight just before Operation } & \multicolumn{4}{|c|}{$\begin{array}{c}\text { Weight One Year after } \\
\text { Operation }\end{array}$} & \multicolumn{4}{|c|}{ Weight at Follow-up $\frac{\sqrt{3}}{\sqrt{3}}$} \\
\hline & & \multicolumn{2}{|c|}{$\begin{array}{l}\text { Duodenal } \\
\text { Ulcer }\end{array}$} & \multicolumn{2}{|c|}{$\begin{array}{l}\text { Gastric } \\
\text { Ulcer }\end{array}$} & \multicolumn{2}{|c|}{$\begin{array}{l}\text { Duodenal } \\
\text { Ulcer }\end{array}$} & \multicolumn{2}{|c|}{$\begin{array}{l}\text { Gastric } \\
\text { Ulcer }\end{array}$} & \multicolumn{2}{|c|}{$\begin{array}{l}\text { Duodenal } \\
\text { Ulcer }\end{array}$} & \multicolumn{2}{|c|}{$\begin{array}{l}\text { Gastric } \\
\text { Ulcer }\end{array}$} & \multicolumn{2}{|c|}{$\begin{array}{l}\text { Duodenal } \\
\text { Ulcer }\end{array}$} & \multicolumn{2}{|c|}{ 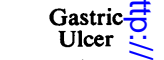 } \\
\hline & & Male & $\begin{array}{c}\mathrm{Fe}- \\
\text { male }\end{array}$ & Male & $\begin{array}{c}\mathrm{Fe}- \\
\text { male }\end{array}$ & Male & $\begin{array}{c}\mathrm{Fe}- \\
\text { male }\end{array}$ & Male & $\begin{array}{c}\mathrm{Fe}- \\
\text { male }\end{array}$ & Male & $\begin{array}{c}\mathrm{Fe}- \\
\text { male }\end{array}$ & Male & $\begin{array}{c}\text { Fe- } \\
\text { male }\end{array}$ & Male & $\begin{array}{c}\text { Fe- } \\
\text { male }\end{array}$ & Male & $\begin{array}{l}\text { Fe } \\
\text { mate } \\
\text { de }\end{array}$ \\
\hline \multirow[t]{4}{*}{1 and 2} & \multirow{4}{*}{$\begin{array}{l}\text { Above ideal weight } \\
20 \% \text { or more } \\
10 \% \\
\text { No difference } \\
\text { Below ideal weight } \\
10 \% \text { o } \\
20 \% \text { or more } \\
\text { Mean weight } \\
\text { Number of cases } \\
\text { weight known } \\
\text { Number of cases } \\
\text { weight not known }\end{array}$} & $\begin{array}{l}11 \cdot 8 \\
23 \cdot 1 \\
42.0\end{array}$ & $\begin{array}{l}22.7 \\
13.6 \\
18.2\end{array}$ & $\begin{array}{r}5 \cdot 5 \\
26 \cdot 8 \\
39 \cdot 4\end{array}$ & $\begin{array}{r}9 \cdot 0 \\
33 \cdot 3 \\
12 \cdot 2\end{array}$ & $\begin{array}{r}4.9 \\
10.9 \\
32.7\end{array}$ & $\begin{array}{r}6 \cdot 6 \\
4 \cdot 4 \\
22 \cdot 2\end{array}$ & $\begin{array}{r}0.8 \\
4.0 \\
25.4\end{array}$ & $\begin{array}{r}2.9 \\
11.8 \\
11.8\end{array}$ & $\begin{array}{r}3.1 \\
9.0 \\
27.5\end{array}$ & $\begin{array}{r}7 \cdot 3 \\
4 \cdot 9 \\
12 \cdot 2\end{array}$ & $\begin{array}{r}1.6 \\
8.1 \\
29.8\end{array}$ & $\begin{array}{l}\overline{13 \cdot 3} \\
16 \cdot 7\end{array}$ & $\begin{array}{r}5 \cdot 2 \\
12 \cdot 0 \\
26 \cdot 6\end{array}$ & $\begin{array}{r}8.9 \\
6.7 \\
11 \cdot 1\end{array}$ & $\begin{array}{r}1.6 \\
8.5 \\
31.0\end{array}$ & $\frac{3}{3 .}$ \\
\hline & & $\begin{array}{r}20 \cdot 1 \\
3.0 \\
+2.3\end{array}$ & $\begin{array}{r}25.0 \\
20.5 \\
+0.5\end{array}$ & $\begin{array}{r}24.4 \\
3.9 \\
+0.7\end{array}$ & $\begin{array}{r}27.3 \\
18.2 \\
-0.9\end{array}$ & $\begin{array}{r}33.1 \\
18.4 \\
-4.9\end{array}$ & $\begin{array}{r}13.3 \\
53.4 \\
-12.9\end{array}$ & $\begin{array}{r}46 \cdot 1 \\
23 \cdot 8 \\
-9 \cdot 2\end{array}$ & $\begin{array}{r}11.8 \\
61.7 \\
-14.7\end{array}$ & $\begin{array}{r}37.3 \\
23.2 \\
-6.9\end{array}$ & $\begin{array}{r}22.0 \\
53.6 \\
-12.2\end{array}$ & $\begin{array}{r}39 \cdot 5 \\
20.9 \\
-7 \cdot 2\end{array}$ & $\begin{array}{r}23 \cdot 3 \\
46 \cdot 7 \\
-12.0\end{array}$ & $\begin{array}{r}26 \cdot 7 \\
19.5 \\
-5.4\end{array}$ & $\begin{array}{r}24.4 \\
48.9 \\
-10.9\end{array}$ & $\begin{array}{r}38 \cdot 0 \\
20 \cdot 9 \\
-7 \cdot 1\end{array}$ & $\begin{array}{r}176 \\
4401 \\
-106\end{array}$ \\
\hline & & 264 & 44 & 127 & 33 & 266 & 45 & 126 & 34 & 255 & 41 & 124 & 30 & 267 & 45 & 129 & \\
\hline & & 13 & 3 & 7 & 5 & 11 & 2 & 8 & 4 & 22 & 6 & 10 & 8 & 10 & 2 & 5 & \\
\hline \multirow[t]{4}{*}{3} & \multirow{4}{*}{$\begin{array}{l}\text { Above ideal weight } \\
20 \% \text { or more } \\
10 \% \text { orfence } \\
\text { No diference } \\
\text { Below ideal weight } \\
10 \% \text {. } \\
20 \% \text { or more } \\
\text { Mean weight } \\
\text { Number of cases } \\
\text { weight known } \\
\text { Number of cases } \\
\text { weight not known }\end{array}$} & $\begin{array}{r}9 \cdot 6 \\
32 \cdot 9 \\
35 \cdot 6\end{array}$ & $\begin{array}{l}14.8 \\
25.9 \\
18.5\end{array}$ & $\begin{array}{r}4.8 \\
19.5 \\
36.6\end{array}$ & $\begin{array}{l}10 \cdot 0 \\
20 \cdot 0 \\
25 \cdot 0\end{array}$ & $\begin{array}{r}\overline{8.5} \\
36.6\end{array}$ & $\overline{\overline{15.4}}$ & $\begin{array}{r}\overline{7 \cdot 3} \\
17 \cdot 1\end{array}$ & $\overline{\overline{10}}$ & $\begin{array}{r}\overline{4 \cdot 3} \\
27 \cdot 1\end{array}$ & $\begin{array}{r}\overline{13.0} \\
4 \cdot 3\end{array}$ & $\begin{array}{l}2.5 \\
5.0 \\
5.0\end{array}$ & 二 & $\begin{array}{r}1.4 \\
2.7 \\
24.3\end{array}$ & $\begin{array}{r}3.7 \\
3.7 \\
14.8\end{array}$ & $\begin{array}{r}\overline{2 \cdot 4} \\
12 \cdot 2\end{array}$ & $\frac{N}{\frac{N}{\omega}}$ \\
\hline & & $\begin{array}{r}20.5 \\
1.4 \\
+2.9\end{array}$ & $\begin{array}{r}25.9 \\
14.8 \\
+1.1\end{array}$ & $\begin{array}{r}34.1 \\
4.9 \\
-0.7\end{array}$ & $\begin{array}{r}20.0 \\
25.0 \\
-3.0\end{array}$ & $\begin{array}{r}42 \cdot 3 \\
12 \cdot 7 \\
-6 \cdot 2\end{array}$ & $\begin{array}{r}26.9 \\
57.7 \\
-15.8\end{array}$ & $\begin{array}{r}29 \cdot 3 \\
46.3 \\
-12.2\end{array}$ & $\begin{array}{r}35.0 \\
55.0 \\
-18.0\end{array}$ & $\begin{array}{r}42.9 \\
25 \cdot 8 \\
-9.3\end{array}$ & $\begin{array}{r}26.1 \\
56.4 \\
-15.7 \\
\end{array}$ & $\begin{array}{r}40.0 \\
47.5 \\
-13.5\end{array}$ & $\begin{array}{r}31 \cdot 6 \\
68.5 \\
-21.1\end{array}$ & $\begin{array}{r}50.0 \\
21.6 \\
-9.2\end{array}$ & $\begin{array}{r}18.5 \\
59.2 \\
-13.7\end{array}$ & $\begin{array}{r}29.3 \\
56.0 \\
-14.6\end{array}$ & $\begin{array}{r}300 \\
700 \\
-2 \notin 55\end{array}$ \\
\hline & & 73 & 27 & 41 & 20 & 71 & 26 & 41 & 20 & 70 & 23 & 40 & 19 & 74 & 27 & 41 & \\
\hline & & 1 & - & 1 & - & 3 & 1 & 1 & - & 4 & 4 & 2 & 1 & - & - & 1 & 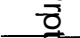 \\
\hline \multirow[t]{4}{*}{$\overline{4 \text { and } 5}$} & \multirow{4}{*}{$\begin{array}{l}\text { Above ideal weight } \\
20 \% \text { or more } \\
10 \% \\
\text { No difference } \\
\text { Below ideal weight } \\
10 \% \\
20 \% \text { or more } \\
\text { Mean weight } \\
\text { Number of cases } \\
\text { weight known } \\
\text { Number of cases } \\
\text { weight not known }\end{array}$} & $\begin{array}{r}6.0 \\
26.0 \\
52.0\end{array}$ & $\begin{array}{l}14 \cdot 3 \\
14 \cdot 3 \\
42 \cdot 9\end{array}$ & $\begin{array}{r}5.0 \\
20.0 \\
40.0\end{array}$ & $33 \cdot 3$ & $\begin{array}{r}7 \cdot 0 \\
40 \cdot 0\end{array}$ & $\overline{12.5}$ & $\begin{array}{r}5 \cdot 3 \\
36 \cdot 8\end{array}$ & $\overline{\overline{16} \cdot 7}$ & $\begin{array}{r}\overline{2 \cdot 1} \\
29 \cdot 2\end{array}$ & 二 & $\overline{\overline{5} \cdot 3}$ & 二 & $\begin{array}{r}\overline{4 \cdot 0} \\
22 \cdot 0\end{array}$ & $\overline{\overline{14} \cdot 3}$ & $\begin{array}{l}\frac{4 \cdot 5}{二} \\
-\end{array}$ & $\frac{\frac{O}{0}}{\frac{0}{0}}$ \\
\hline & & $\begin{array}{r}10.0 \\
6.0 \\
+1.6\end{array}$ & $\begin{array}{r}\overline{28.6} \\
-1.4\end{array}$ & $\begin{array}{r}25.0 \\
10.0 \\
-1.5\end{array}$ & $\begin{array}{r}33 \cdot 3 \\
33 \cdot 3 \\
-6 \cdot 7\end{array}$ & $\begin{array}{r}36.0 \\
18.0 \\
-1.7\end{array}$ & $\begin{array}{r}25.0 \\
25.0 \\
-7.5\end{array}$ & $\begin{array}{r}26.3 \\
31.6 \\
-8.9\end{array}$ & $\begin{array}{r}16.7 \\
66.7 \\
-1.5\end{array}$ & $\begin{array}{r}37.5 \\
31 \cdot 3 \\
-9.8\end{array}$ & $\begin{array}{r}25.0 \\
62.5 \\
-21.3\end{array}$ & $\begin{array}{r}31.6 \\
63.1 \\
-16.8\end{array}$ & $\begin{array}{r}40.0 \\
60.0 \\
-18.0\end{array}$ & $\begin{array}{r}48.0 \\
26.0 \\
-9.8\end{array}$ & $\begin{array}{r}14.3 \\
71.4 \\
-15.7\end{array}$ & $\begin{array}{r}27 \cdot 3 \\
68 \cdot 2 \\
-17.7\end{array}$ & $\begin{array}{r}167 \\
88_{3} \\
-2 \underbrace{}_{3}\end{array}$ \\
\hline & & 50 & 7 & 20 & 6 & 50 & 8 & 19 & 6 & 48 & 8 & 19 & 5 & 50 & 7 & 22 & •̈. \\
\hline & & 2 & 1 & 3 & 1 & 2 & - & 4 & 1 & 4 & - & 4 & 2 & 2 & 1 & 1 & \\
\hline
\end{tabular}


good (groups 1 and 2) and unsatisfactory (groups 4 and 5) operation results and represented them in graph form (Figs. 2 and 3).

From the figures presented the following facts emerge:

(a) The mean weights in both sexes at the time of operation were considerably below the "best ever" weights, but here other factors such as age may be responsible as well as the ulcer. This does not appear to apply in groups 4 and 5 results for gastric ulcer in women but the actual number of cases is so small that the figures are not significant.

(b) The mean weights in both sexes at the time of operation in all sub-groups were below the standard weight and very few individual cases were above standard weight.

(c) Patients who had achieved a good result from operation by the basic assessment appeared on the average to maintain or slightly increase their weight after operation, but the mean weights were still below the standard weights.

(d) Those who had an unsatisfactory result from operation failed on the average to maintain their pre-operative weight. The majority of weight losses in these patients appeared to be in the first year after operation.

(e) It may be of interest to note that the mean weights of groups 4 and 5 result cases in all subgroups were slightly higher before operation than those of groups 1 and 2 result cases.

\section{FaILURES AFTer GASTRECTOMY}

Of the 826 cases followed up, 99 (12\%) were not considered sufficiently satisfactory for one reason or another and have been classified as failures. This group of 99 patients consists of group 4 and group 5 results. In group 5 either further surgical or medical treatment had been required or the patient had expressed his or her dissatisfaction with the operation. In group 4, patients on the whole preferred their present state to the original ulcer but had in the investigators' opinion symptoms which prevented them leading a normal life. In the tables in which results of operation are compared with the series of factors much information is given regarding the group 4 and 5 cases. It is considered sufficiently interesting, however, to discuss these failures in a little more detail.

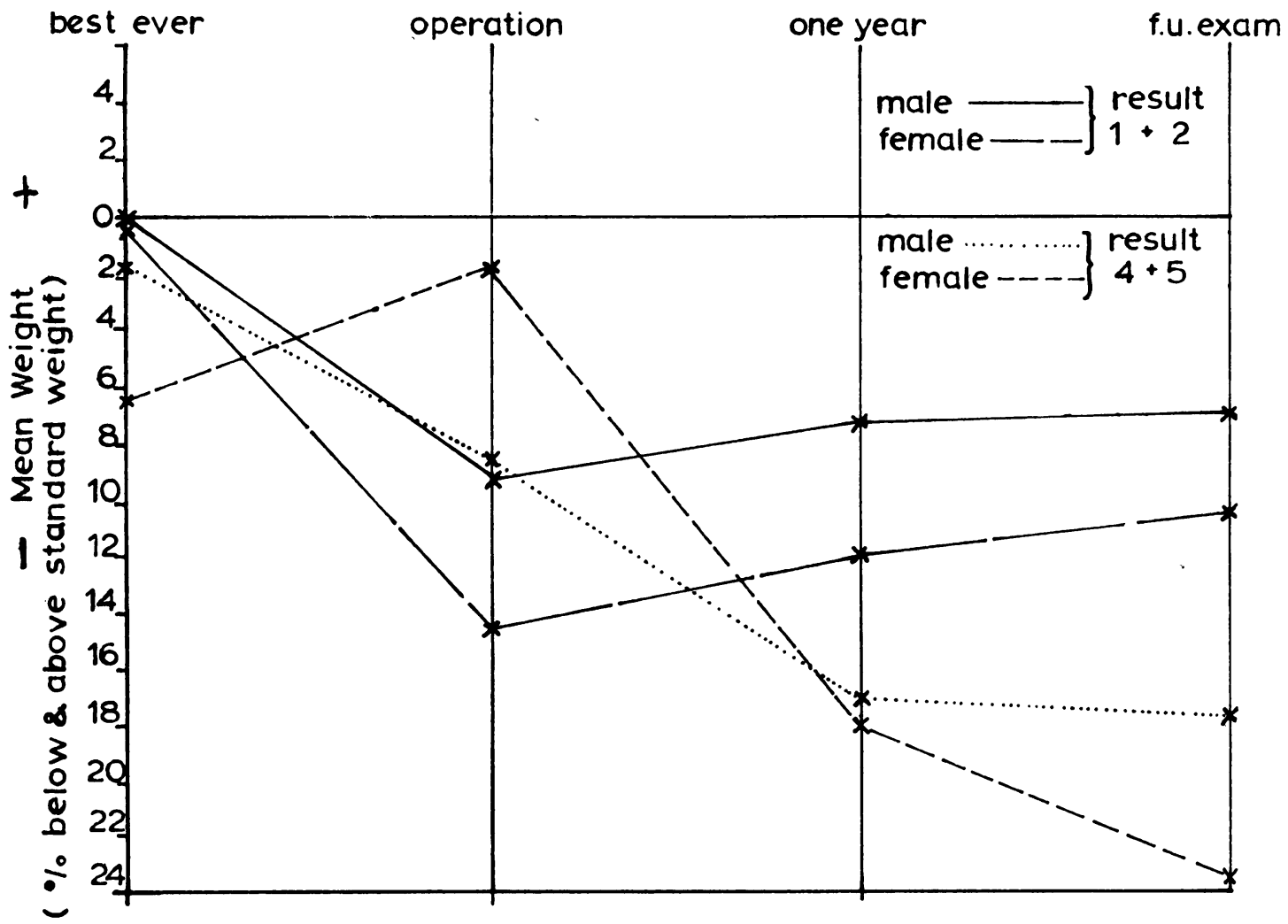

FIG. 2.-Mean weights for both sexes for good and for unsatisfactory groups in duodenal ulcer cases. 


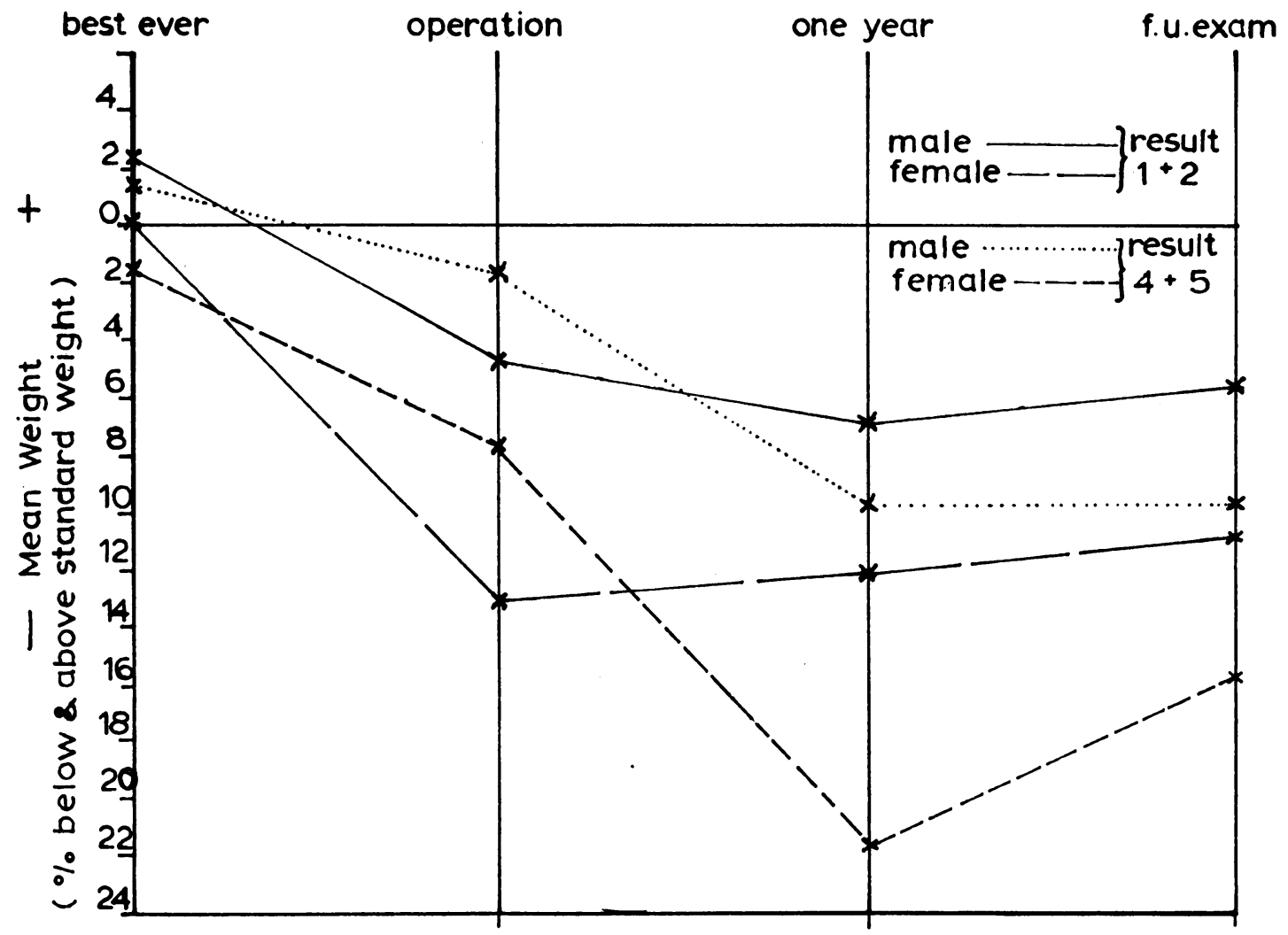

FIG. 3.-Mean weights for both sexes for good and for unsatisfactory groups in gastric ulcer cases.

Group 5 Patients. - There were 57 patients in group $5,6.9 \%$ of the total followed up. They are considered in the following sub-groups according to the chief symptoms or cause of failure (Table XVI).

Bilious Vomiting: Afferent Loop Syndrome.Seventeen cases were classed as failures because of severe and persistent bilious vomiting, sometimes associated with other features of the afferent loop syndrome, and needed a further operation. In 12 cases the symptoms came on very soon after the original gastrectomy and only in one case was the onset later than 18 months. All occurred after a Polya type operation and in seven of the patients a Hofmeister modification of that operation had been done.

TABLE XVI

ANALYSIS OF GROUP 5 RESULTS

\begin{tabular}{|c|c|c|c|c|c|c|c|c|c|c|c|c|}
\hline \multirow[b]{2}{*}{ Causes of Failure } & \multirow[b]{2}{*}{$\begin{array}{l}\text { Total } \\
\text { No. }\end{array}$} & \multicolumn{5}{|c|}{ Original Operation } & \multicolumn{4}{|c|}{ Original Ulcer } & \multicolumn{2}{|c|}{ Sex } \\
\hline & & $\underset{\text { Pelrocolic }}{\text { Rolya }}$ & $\begin{array}{l}\text { Antecolic } \\
\text { Polya }\end{array}$ & $\begin{array}{c}\text { Antecolic } \\
\text { Polya } \\
\text { Hofmeister }\end{array}$ & $\begin{array}{c}\text { Retrocolic } \\
\text { Polya } \\
\text { Hofmeister }\end{array}$ & Billiroth & $\begin{array}{c}\text { Duodenal } \\
\text { Ulcer }\end{array}$ & $\begin{array}{l}\text { Gastric } \\
\text { Ulcer }\end{array}$ & $\begin{array}{c}\text { Duodenal } \\
\text { and } \\
\text { Gastric } \\
\text { Ulcer }\end{array}$ & $\begin{array}{c}\text { Stomal } \\
\text { Ulcer } \\
\text { after } \\
\text { Gastro- } \\
\text { enterostomy }\end{array}$ & Male & $\begin{array}{r}\text { Femaled } \\
\frac{\mathrm{C}}{\mathrm{D}}\end{array}$ \\
\hline $\begin{array}{l}\text { Bilious vomiting } \\
\text { Faintness, sweating, }\end{array}$ & 17 & - & 9 & 7 & 1 & - & 13 & 3 & 1 & - & 12 & $5 \frac{\sqrt[3]{8}}{8}$ \\
\hline $\begin{array}{l}\text { diarrhoea } \\
\text { Stomal ulcer }\end{array}$ & $\begin{array}{r}2 \\
15\end{array}$ & 二 & $\begin{array}{l}1 \\
8\end{array}$ & $\begin{array}{l}1 \\
4\end{array}$ & $\overline{-}$ & $\overline{3}$ & 25 & 二 & 二 & $\overline{2}$ & 2 & $-\frac{\sigma}{<}$ \\
\hline $\begin{array}{l}\text { Steatorrhoea } \\
\text { Miscellaneous com- }\end{array}$ & 3 & - & 3 & - & - & - & 3 & - & - & - & 3 & -8 \\
\hline $\begin{array}{l}\text { plications } \\
\text { Patient's verdict of }\end{array}$ & 3 & - & 3 & - & - & - & 2 & 1 & - & - & 3 & 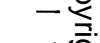 \\
\hline "failure" & 17 & - & 11 & 5 & - & 1 & 8 & 8 & 1 & - & 16 & 1 \\
\hline Total & 57 & - & 35 & 17 & 1 & 4 & 41 & 12 & 2 & 2 & 50 & 7 \\
\hline
\end{tabular}


The second, or "corrective", operation was enteroenterostomy in eight cases, Polya to Billroth conversion in seven cases, and division of adhesions in one case only. Of the entero-enterostomies, seven patients have since improved sufficiently to be now equivalent to group 1 or 2 results and the other to group 4. Of the Polya to Billroth conversions, three are equivalent to group 1 or 2 results, one to group 3, and one to group 4, with two cases too early for assessment. In one case only were adhesions divided and that patient is only slightly improved. The remaining patient is of interest in that two years after operation he developed a rupture of the duodenum due to afferent loop distension. The duodenum was repaired as an emergency. He still has bilious vomiting and is awaiting further surgery for this.

Faintness, Sweating after Meals, and Severe Diarrhoea.-Two patients had this combination of symptoms after Polya gastrectomy and a Henley loop type of gastroplasty was subsequently carried out in both. Neither case subsequently showed any improvement.

Steatorrhoea.-Three cases were classified as failures because of the development of steatorrhoea after operation. In all three the steatorrhoea was severe enough to be incapacitating and had been proved by hospital investigation. All were men and steatorrhoea had followed antecolic Polya operations.

Stomal Ulcer.-Fifteen cases were considered to have developed stomal ulcer since operation. In 12 of these the diagnosis was based on the findings at a second operation, radiographs, or on evidence of post-gastrectomy intestinal haemorrhage. The other three patients had had ulcer-type symptoms very suggestive of recurrent ulcer.

Of the 15 patients, 13 had had gastrectomy for duodenal ulcer and the other two had had gastrectomy for a stomal ulcer following a previous gastroenterostomy for duodenal ulcer. Twelve of them occurred in patients who had had a Polya type operation and the other three had had Billroth gastrectomies. All except one were in men.

Re-operation was carried out in 10 cases, consisting of vagotomy in seven, conversion from Polya to Billroth in one, Henley loop gastroplasty in one, and extension of Polya gastrectomy in the other. Two cases after vagotomy are now symptomfree and one is improved. The case converted to Billroth improved slightly and is now equivalent to group 3, while the case after Henley loop gastroplasty was again a failure and needed excision of the jejunal loop. Other cases are too early to assess results.
Miscellaneous.-In three patients failure occurred but was perhaps not of the type usually classed as post-gastrectomy syndrome. In all three, however, it was considered that the original gastrectomy was indirectly responsible for their present incapacity. One had pulmonary tuberculosis before gastrectomy and this became much worse immediately after the operation. In the second the common bile duct was damaged surgically at the time of operation and the patient has had recurrent cholangitis since then. The third developed severe peritoneal infection after the operation which led to extensive adhesions and has needed four subsequent operations for intestinal obstruction with much disability. All three were men. These failures followed antecolic Polya operations. In two the original ulcer was duodenal, while the other had a gastric ulcer.

Patient's Verdict of "Failure".-_Seventeen patients at interview stated that their present condition was worse than that before gastrectomy and that they were sorry they had had the operation performed. Their symptoms and complaints were too varied and multiple to classify into any particular group. Faintness after meals, inability to take large meals, lassitude, occasional bilious vomiting, abdominal discomfort after meals, loss of appetite, and nausea were the most frequent symptoms. One patient had had a subsequent Henley loop gastroplasty performed without improvement. Another had needed an operation for volvulus of the gastric stump. Four patients had needed subsequent psychiatric treatment for depressive psychoses. In none was the clinical picture of the symptoms, nor, in some, the results of investigations sufficient to warrant further surgery. Furthermore, in this group on the whole, the patients voiced the opinion that they would not consider further operation even if it were raised. It was our impression that possibly the fault in these patients lay in original misjudgments in selecting cases for gastrectomy.

In this group 16 of the $\mathbf{1 7}$ failures were in men; eight followed operations for gastric ulcer, one for gastric and duodenal ulcer, and eight for duodenal ulcer. The proportion of men and of gastric ulcer types is higher in this group than in the total series. Sixteen of these patients had had antecolic Polya operations, five of them of the Hofmeister modification. One patient had had a Billroth gastrectomy.

Group 4 Patients. - In group 4 there were 42 patients, representing $5 \cdot 1 \%$ of the total followed up. None, of course, had required further surgery as far as the gastrectomy was concerned, none had recurrent ulcers, and none of the patients had expressed complete dissatisfaction with the opera- 
TABLE XVII

ANALYSIS OF GROUP 4 RESULTS

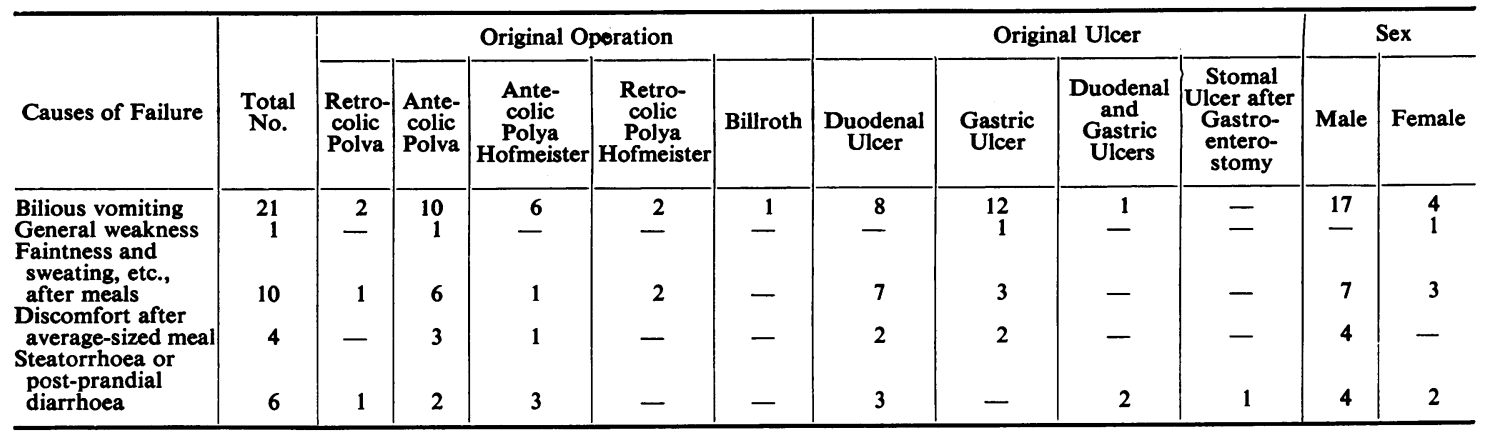

tion. These factors would have made them group 5 results. In our original analysis we had classified the patients according to their chief post-gastrectomy symptoms rather than to particular groups of symptom complexes. We did this because we found so many cases in which the patient's particular collection of symptoms did not always fit into any one set post-gastrectomy syndrome. In Table XVII, analysing group 4 results, we have classed the patients according to the main symptoms of which they complained. Hence 21 of the 42 patients in this group complained chiefly of bilious vomiting although some of them also had faintness or abdominal discomfort after meals which did not particularly worry them. Similarly 10 complained chiefly of faintness after meals, four of discomfort after an average-sized meal, and six of steatorrhoea, but some of these also had mild, infrequent bilious vomiting. One of the patients in this group with steatorrhoea had, in fact, got the steatorrhoea before the gastrectomy, since it had followed a gastroenterostomy for duodenal ulcer.

\section{Summary}

A detailed survey has been made of 955 patients in whom partial gastrectomy was performed for peptic ulcer. The operative mortality rate was $3.87 \%$ (37 patients). Of 876 cases available for investigation, $826(94.3 \%)$ were traced and interviewed. After the operation 540 patients $(65.4 \%$ of those followed up) were able to lead a normal life and had no symptoms of any significance, while a further $187(22.6 \%)$ had mild symptoms which needed some dietary care or medical treatment, but which did not prevent them leading a normal life. The remaining 99 were unsatisfactory results and of these $57(6.9 \%)$ patients were as bad as, or worse than, before operation.

The result of the operation did not appear to be influenced by the following factors: (1) Length of follow up, (2) age, (3) site of ulcer, (4) duration of symptoms before operation, or (5) the type of preoperative symptoms. Men appeared to give better results than women to a degree just significant.

The Billroth operation gave better results in gastric ulcer, but for duodenal ulcer that operation gave equally good results to the Polya type. The variations of the Polya operation did not materially alter the results. The extent of gastric resection in both operations did not appear significant, except in men with duodenal ulcer (the largest group) where more limited resections (less than two-thirds) were significantly better.

In the satisfactory results earning capacity was increased and time lost from work reduced. The mean body weight before operation was considerably below the standard weight, and although in the satisfactory patients it showed slight improvement after operation, it still did not approach the standard weight.

The causes of failure in the unsatisfactory cases, some of which improved after further surgery, are analysed.

We should like to express our gratitude to the surgeons and physicians of the Queen Elizabeth Hospital, Birmingham, for allowing us to investigate these cases, and also particularly to Professor Stammers for his encouragement, help, and advice throughout the investigation.

\section{REFERENCES}

Kemsley, W. F. F. (1952), Ann. Eugen. (Lond.), 16, 316. Thorn, P. A., Brookes, V. S., and Waterhouse, J. A. H. (1956). Brit. med. J., 1, 603.

Visick, A. H. (1948a). Lancet, 1, 505.

Visick, A. H. (1948). Ibid., 1, 551. 